\title{
A component-based method for the optimization of stiffener layout on large cylindrical rib-stiffened shell structures
}

\author{
Florent Savine $^{12}$ - François-Xavier Irisarri ${ }^{1}$ - Cédric Julien ${ }^{1}$. \\ Angela Vincenti ${ }^{2}$. Yannick Guerin ${ }^{3}$
}

Received: date / Accepted: date

\begin{abstract}
In the present work, an optimization method is proposed in order to produce innovative stiffening layouts for large stiffened cylindrical shell structures, as they appear in the aerospace industry. A componentbased logic is applied on a ground mesh of structural elements (shells and beams), which was inspired by techniques of explicit topology optimization on solid elements (plane or tridimensional massive models). Geometric components, representing the layout of the stiffeners (i.e. location, shape and size), are projected onto the ground mesh, resulting in controlled sets of active beam elements. These sets constitute the structural representation of the stiffeners' layout in the optimization model, which is then used to evaluate the objective and constraint functions of the optimization problem as well as their semi-analytical sensitivities. By applying the optimization method to compliance minimization problems, we show the efficiency and accuracy of the
\end{abstract}

The authors acknowledge ONERA and CNES for co-funding this project.

Florent Savine

E-mail: florent.savine@onera.fr

François-Xavier Irisarri

E-mail: francois-xavier.irisarri@onera.fr

Cédric Julien

E-mail: cedric.julien@onera.fr

Angela Vincenti

E-mail: angela.vincenti@sorbonne-universite.fr

Yannick Guerin

E-mail: yannick.guerin@cnes.fr

${ }^{1}$ DMAS, ONERA, Université Paris Saclay, Chatillon France

2Sorbonne Université, CNRS, UMR 7190, Institut d'Alembert, Paris - France

${ }^{3}$ CNES, Direction des lanceurs, Paris - France proposed method and its capability to handle a typical aerospace structure, such as a space-launcher part: a stiffened cylindrical shell in presence of an access hatch.

Keywords Stiffener layout optimization · componentbased method · stiffened shell structure · ground structure $\cdot$ explicit topology optimization

\section{Introduction}

Competition in the space industry has drastically increased in the past few years. Industrials are aiming at a massive cost reduction in the design, fabrication and operation of their future generations of launchers. One of the available levers to reduce the costs is to lighten the primary structures of the launchers. On the other hand, these structures withstand massive efforts in compression, locally introduced by the boosters. These efforts can generate stress concentrations which can propagate and damage the structure. To prevent this, the load distribution must be spread within a short distance from the load introduction points. Rib-stiffened structures appear to be a natural solution to these competing mass and structural objectives. However, the most conventional layouts, in rings and stringers or in grid patterns, do not seem optimal when dealing with such localized phenomena. As such, the present work aims at developing an optimization method able to find innovative stiffening layouts when pre-sizing large cylindrical shell structures.

A typical example of the parts that will be dealt with is shown in Figure 1. These stiffened metallic cylindrical parts have a diameter of 5 to $6 \mathrm{~m}$ and a height of 3 to $4 \mathrm{~m}$. They are stiffened by rings and stringers of 100 to $300 \mathrm{~mm}$ high by 1 to $10 \mathrm{~mm}$ thick. Note the great difference in the order of magnitude of the 


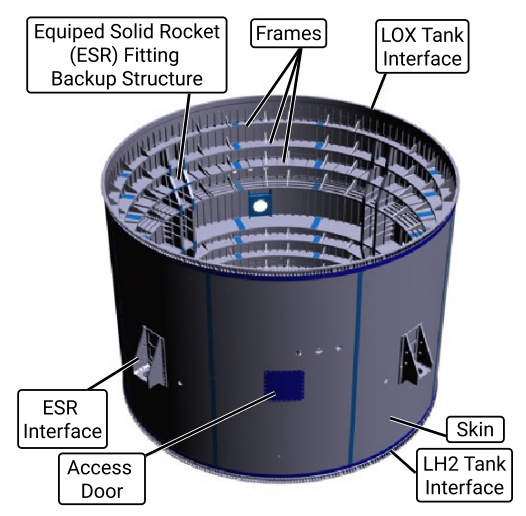

Fig. 1 Intertank Structure (ITS) of the Lower Liquide Prorpulsion Module (LLPM) on the Ariane 6 launcher (Merino et al. 2017).

structural element sizes. This difference leads to modeling the structures using structural elements, i.e. shell elements for the skin of the cylinder and beam elements for the stiffeners. This kind of model has a good performance-to-accuracy ratio and is widely spread in aerospace industry (see Maes et al. (2019) as an example case of launcher structure). Therefore, the proposed optimization method is developed based on the use of structural elements instead of solid elements, which are widely present in the literature when dealing with the optimization of stiffeners' non-regular patterns.

As a matter of fact, the design of stiffened structures has been widely addressed in the literature. One of the main concerns is finding the optimal layout of the stiffeners. Two main strategies can be distinguished. The first strategy consists in parameterizing an initial layout and deforming it through the optimization process, similarly to a sizing optimization. Such a strategy is often applied to grid-stiffened structures as reviewed by Bedair (2009). The variables are usually the spacing between stiffeners disposed in triangular or square patterns. These initial layouts were found to restrict the design domain too much when using composite materials. New initial layouts were introduced like the geodesic (Gurdal and Gendron 1993) or anisogrid (Jaunky 1995; Vasiliev et al. 2001) patterns. Shi et al. (2013) studied the optimization of grid-stiffened structures for conical shells. To better adapt the grid patterns to localized stiffening needs, Wang et al. (2017, 2018) developed a methodology to generate curved grid-stiffened structures. The Finite-Element (FE) models are usually built by smearing the stiffening elements into a plate model with a similar global stiffness by a homogenization process.

Other authors have privileged models with explicit representation of the stiffeners using structural elements.
This allows to consider layouts that are not grid-like. Kapania et al. (2005) were able to model curvilinear stiffeners defined by a spline and moving freely on the surface. This method was then further developed in $(\mathrm{Li}$ et al. 2017c; Mulani et al. 2013; Szczepanik and Burczyński 2012) and made compatible with grid-like patterns (Mulani et al. 2011). Unfortunately, this method necessitates a full remeshing of the structure for each new location of the stiffeners. This is a costly process, especially in the context of very large parts. Mesh-tie techniques (Zhao et al. 2019) can be used to avoid remeshing. Nevertheless, these methods can be intrusive in the finite element code, especially for the derivation of sensitivities. Hirschler et al. (2019) propose an optimization method which solves many of the aforementioned issues in the isogeometric framework.

The second strategy to find the optimal layout of the stiffeners is to deduce the stiffening scheme from a shape or topology optimization result. Using topology optimization, Niemann et al. (2013) optimized a complete fuselage section and proposed an innovative stiffener layout issued from the resulting skin thickness distribution. However, topology optimization results are usually difficult to interpret as realistic stiffening structures and a secondary optimization step is often conducted in order to get a structure that is feasible with respect to manufacturing processes. In any cases, retrieving a consistent CAD from a topological optimization result is a difficult task. Dugré (2014) interprets each discernible member to better constrain a second topology optimization. Other methods (Afonso et al. 2005; Lam and Santhikumar 2003; Liu et al. 2016) consist in placing stiffening ribs on the high density zones and running a sizing optimization on them. Luo and Gea (1998) used a second optimization step to find the optimal material orientations. Finally, Liu et al. (2015) manage to obtain a stiffener-like topological shape by doing the topology optimization with $3 \mathrm{D}$ elements and casting process constraints. To facilitate the post-processing phase, methods using explicitly defined components have been developed, as reviewed by Wein et al. (2020). Two research groups (Guo et al. 2014; Zhang et al. 2018, 2017) and (Norato et al. 2015; Zhang and Norato 2017) have extensively developed componentbased methods applied to topology optimization, respectively the Moving Morphable Components (MMC) and the Geometry Projection (GP). These methods were generalized into a Generalized Geometry Projection (GGP) tool by Coniglio et al. (2020). Hollow structures, which can be considered as stiffeners with more complex cross-sections, were also considered (Bai and Zuo 2020) using the MMC. Finally a Moving Morphable Voids (MMV) method (Zhang et al. 2020b,c) adapts the 
component-based logic to optimize void regions parametrized by B-spline curves. The drawback of these methods is that a very refined mesh of $3 \mathrm{D}$ solid elements is required in order to accurately represent the stiffeners. Hence, the computation cost soars when these methods are applied to large structures, as we consider in the present work. To reduce these computation costs, Zhang et al. (2020a) have developed an adaptive mesh refinement technique. To avoid using $3 \mathrm{D}$ solid elements to mesh the stiffeners, Sun et al. (2020) propose to smear the stiffeners defined as components in the MMC approach.

Alternative methods (Ding and Yamazaki 2005; Li et al. 2013; Xue et al. 2012) propose to make the stiffening network grow within a ground structure made of beam elements. The beam elements, placed in between every nodes of each shell element, initially have a negligible cross section. From user defined sprouting points, beam elements are added to the stiffening structure by increasing their cross-sections. However, the ground structure constrains the directions of the stiffeners to a limited set of directions. Hence, Li et al. (2017a,b, 2019) developed a stiffness-transformation operation in order to free the stiffening structure from the ground mesh, based on a method which is similar to the stiffness spreading method developed for truss structures (Wei et al. 2014; Cao et al. 2018): akin to smeared methods, the stiffness matrix of the stiffeners, defined as shell elements, are assembled to the stiffness matrix of the surrounding shell elements of the panel.

In the present paper, we propose to apply the logic of the component-based methods to a ground mesh of structural elements. The novelty of the method is to represent the stiffeners as components that are projected on a controlled set of beam elements from the ground structure in order to build the FE model over which the optimization functions, objective and constraints, are evaluated. This method hence benefits from the ease of interpretation of the component method in terms of stiffening patterns, associated to the cost efficiency of using structural FE elements for both the skin and stiffeners. By updating the set of beam elements for each new stiffener location, the stiffener is allowed to freely move over the entire surface without need for remeshing. Furthermore, semi-analytical sensitivities can be derived which enables gradient-based optimization.

The bulk of the paper is organized as follows. In Section 2 the method used to project the stiffener component into a set of beam elements is explained. The optimization process and the derivation of the sensitivities are presented in Section 3. Section 4 details the calibration of the projection functions to ensure the ac- curacy of the projected model. In Section 5 we apply the proposed method to a cantilever plate and two large cylindrical structures with and without an access hatch. Finally, some conclusions on the method are drawn in Section 6.

\section{Component-based representation and projection onto the FE model}

The method presented in this paper is inspired by featuremapping methods: a geometrical representation of the stiffeners (position, layout, size) is projected onto a FE mesh of the ground structure to build a structural representation of the stiffeners for analysis. This section describes in details both the geometrical and the structural representation, as well as the projection method used to build the FE model of the stiffener from the geometrical description of the components. In the following, for sake of simplicity, the method is illustrated considering the case of a single stiffener on a flat panel.

\subsection{Geometrical representation of the stiffener: a component}

The geometrical representation of the stiffener can be viewed as a simplified CAD representation, where a stiffener is represented by a component. The component is visualized as a line segment and has associated material and cross-sectional properties. The location of the component is parametrized by the coordinates of the two extremities $\mathrm{P}_{1}$ and $\mathrm{P}_{2}$ of the line segment: $x_{1}, y_{1}$, $x_{2}$ and $y_{2}$, measured with respect to the global reference frame of the structure. A component has also specified cross-sectional properties: the area $A_{c}$, the second moment inertias $I_{y_{c}}$ and $I_{z c}$, the torsional constant $J_{c}$ and the section offset $h_{c}$ (see Figure 2). These properties can advantageously be derived from any stiffener crosssection geometry (blade, hat, Z, etc.). We consider that the stiffener is made of a homogeneous isotropic linear elastic material, thus the material properties associated to a component are the Young's modulus $E$, the Poisson ratio $\nu$ and the material density $\phi$.

\subsection{FE representation of the ground structure}

The structural representation of the stiffener is obtained by a projection of the component over the FE mesh of the ground structure, which is composed of shell and beam elements. In order to build the FE mesh of the ground structure, one has to start from the support 
panel: its reference surface (mid-surface) is meshed using quadrangular shell elements. Then, beam elements are placed in between every adjacent shell nodes, diagonals included (see Figure 2), and have inertia properties aligned with their respective axis. However, this grid of beam elements must not affect the effective response of the panel: their cross-sectional properties are initially set to very low values, so that their contribution to the overall stiffness of the structure is negligible. Ideally, the shell elements are square, leading to only four possible beam orientations : $0^{\circ}, 90^{\circ}, 45^{\circ}$ and $-45^{\circ}$. The FE mesh of the ground structure is built once and for all, based on the geometrical domain occupied by the panel to be stiffened: thus, our method needs no remeshing step and only the cross-sectional properties of the beam elements are updated in order to represent the layout of the stiffeners over the structure. This is obtained by properly tuning projection functions, which are introduced in the next paragraph.

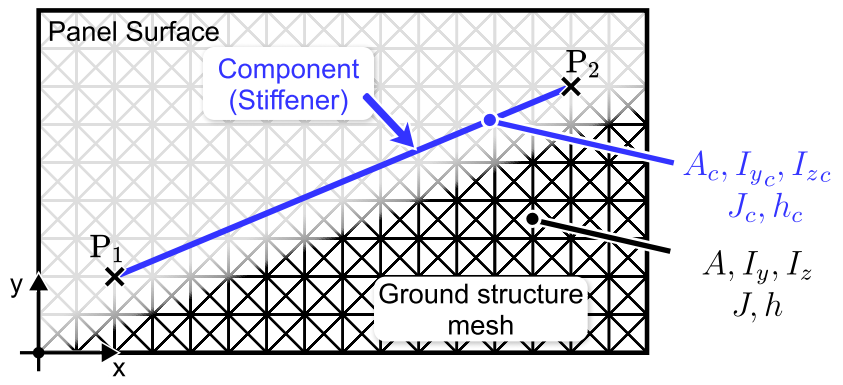

Fig. 2 Superposition of the component model and of the ground structure mesh with $\left\{A_{c}, I_{y_{c}}, I_{z_{c}}, J_{c}, h_{c}\right\}$ and $\left\{A, I_{y}, I_{z}, J, h\right\}$ the cross-sectional properties of a component and of a beam element respectively.

2.3 Structural representation of the stiffener:

projection functions

The major idea of this paper is to approximate the stiffener, defined at the component level by its length and cross-sectional properties, by a set of beam elements from the ground structure. Figure 3 shows a component and the corresponding set of beam elements. Whenever the position of the component changes, the set is updated accordingly by modifying the cross-sectional properties of the beam elements. The principle is that beam elements which are close and well aligned to the component have assigned higher values of cross-sectional properties, whilst the cross-sectional properties decrease when getting far from the component. Hence, the mesh remains fixed while the component may move and rotate freely on the surface.

In order to select the set of beam elements from the ground structure for the structural representation of the stiffener, "projection functions" are used, which establish the updated values of the cross-sectional properties of the beam elements as a fraction of the cross-sectional properties of the component presented in Figure 2. The result of the projection is an updated structural model ready for finite element analysis (see Figure 3 ). The projections functions $\phi^{(P)}$ establish the cross-sectional properties $P=\left\{A, I_{y}, I_{z}, J, h\right\}$ (area, inertia, etc. ) of the beam elements with respect to the corresponding cross-sectional properties $P_{c}$ of the component as follows :

$P=\phi^{(P)} \cdot P_{c}$

Each projection function $\phi^{(P)}$ is tailored for each property $\mathrm{P}$ but they all share the same general form. Their values ranging between 0 and 1 are determined by the product of three filters :

$\phi^{(P)}=f_{a} \cdot f_{d}^{(P)} \cdot f_{l}$

where $f_{a}$ is an angle filter, $f_{d}^{(P)}$ is a distance filter and $f_{l}$ a location filter, written in the form of Gaussian functions. Whilst the angle filter $f_{a}$ and the location filter $f_{l}$ are the same for all cross-sectional properties, the distance filter $f_{d}^{(P)}$ is adapted specifically for each property $P$, as we will explain in the following. In order to build the FE structural model illustrated in Figure 3, filter functions are evaluated for each beam element from the ground structure model as well as the resulting projection function Eq.(2), so that the cross-sectional properties of every beam element are set according to expression Eq.(1).

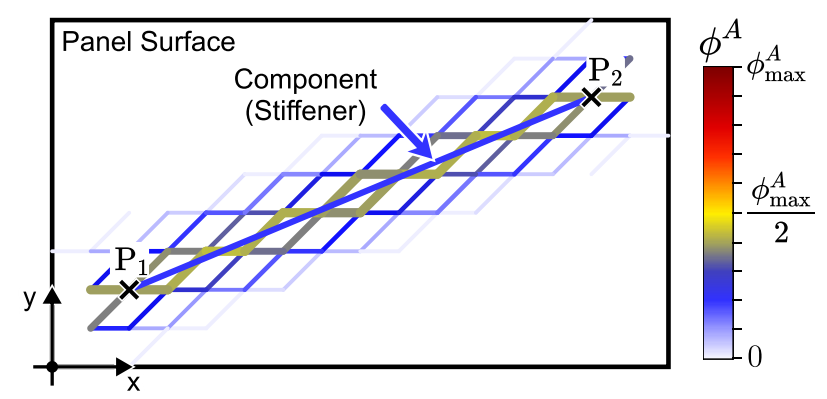

Fig. 3 Structural model of the projected component model of Figure 2. The beam elements are colored according to their projected cross-sectional area $\phi^{A}$. 


\subsubsection{Angle filter $f_{a}$}

The angle filter $f_{a}$ cancels out the elements that are not closely aligned with the component. It is defined as :

$f_{a}\left(\gamma_{i}\right)=\exp \left(\frac{-\gamma_{i}^{2} \ln (2)}{(\pi / 8)^{2}}\right)$

where $\gamma_{i}$ is the angle between the $i$-th beam element and the component (see Figure 4). As such, function $f_{a}$ is maximum and equals one when $\gamma_{i}=0$, and decreases when the beam element orientation deviates. The parameters of the angle filter are chosen so that when the component is the bisector of elements at $0^{\circ}$ and $45^{\circ}$, i.e. $\gamma_{i}=22.5^{\circ}$, the angle filter will have a value $f_{a}=0.5$. Beam elements forming an angle $\left|\gamma_{i}\right|>45^{\circ}$ with the component will have a filter value $f_{a}<0.06$, and will thus be filtered out.

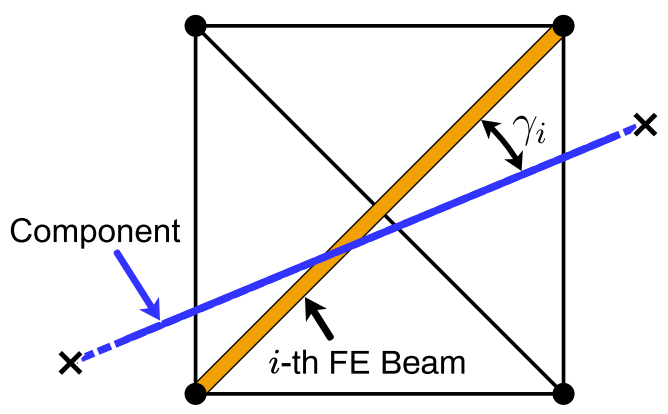

Fig. 4 Definition of the angle $\gamma_{i}$ in the angle filter $f_{a}$.

\subsubsection{Distance filter $f_{d}^{(P)}$}

The distance filter $f_{d}^{(P)}$ cancels out the elements that are distant from the stiffener, where the distance is measured at the end-nodes of each beam element. For the generic $i$-th element of the ground structure, if we call $\mathrm{Q}_{i}$ and $\mathrm{R}_{i}$ its end-nodes, the distance filter $f_{d}^{(P)}$ applied to any cross-sectional property $P$ is defined as:

$f_{d}^{(P)}=\sqrt{f_{d_{\text {node }}(P)}^{(P)}\left(d_{\perp}\left(\mathrm{Q}_{i}\right), \theta\right) \cdot f_{d_{\text {node }}}^{(P)}\left(d_{\perp}\left(\mathrm{R}_{i}\right), \theta\right)}$

where the function $d_{\perp}$ is the orthogonal distance between the considered node and the component, and $\theta$ is the angle between the component and a reference axis defining $\theta=0^{\circ}$, commonly chosen as the $x$-axis, as illustrated in Figure 5a. The function $f_{d_{\text {node }}}^{(P)}$ is a nodal distance filter defined as :

$f_{d_{\text {node }}}^{(P)}\left(d_{\perp}, \theta\right)=\phi_{\max }^{(P)} \cdot \exp \left(\frac{-d_{\perp}^{2} \cdot \ln (2)}{\left(a \delta^{(P)}(\theta)\right)^{2}}\right)$

$a$ is the side length of the square shell element, the parameter $\phi_{\max }^{(P)}$ controls the amplitude of the filter and

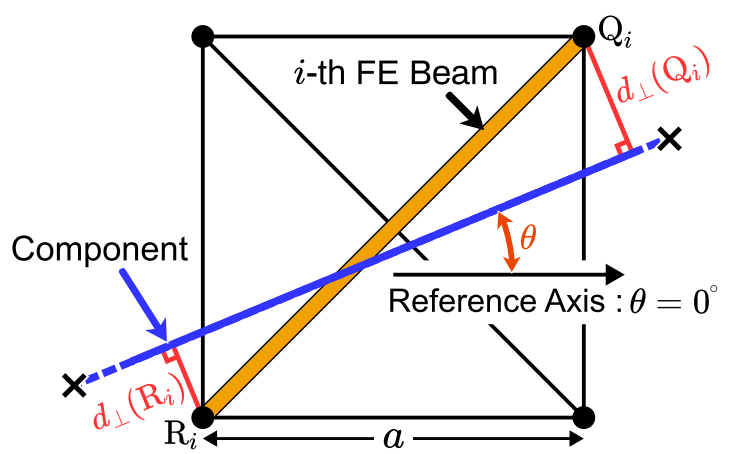

(a)

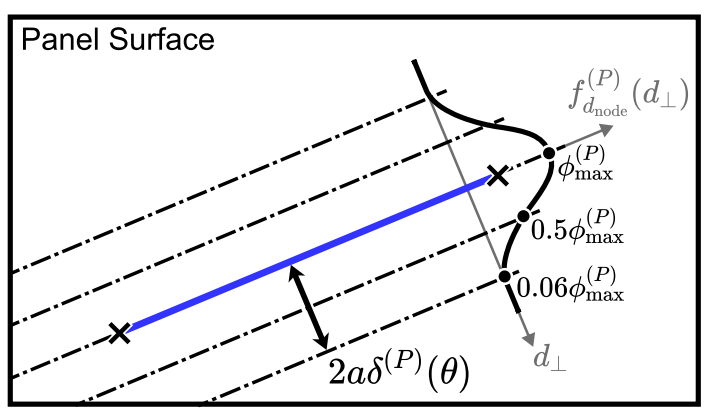

(b)

Fig. 5 (a) Variables of the nodal distance filter $f_{d_{\text {node }}}^{(P)}$. (b) Shape and contour lines of the nodal distance filter $f_{d_{\text {node }}}^{(P)}$ over the panel surface.

the function $\delta^{(P)}(\theta)$ controls its bandwidth, defined as $4 a \delta^{(P)}(\theta)$. These two latter terms, $\phi_{\max }^{(P)}$ and $\delta^{(P)}(\theta)$, are specific to each cross-sectional property $P$. They are the product of a calibration of the filters which is itself based on the resolution of an optimization problem, detailed in Section 4.

From Eq.(5), if the node falls exactly over the component line (i.e. $d_{\perp}=0$ ), the corresponding value of its nodal filter function is $f_{d_{\text {node }}}^{(P)}=\phi_{\max }^{(P)}$ as shown in Figure 5b. For nodes at a distance $d_{\perp}>2 a \delta^{(P)}(\theta)$, the nodal distance filter decreases to $f_{d_{\text {node }}}^{(P)}<0.06 \phi_{\text {max }}^{(P)}$. According to Eq.(4), beam elements that have at least one of their nodes at a distance $d_{\perp}>2 a \delta^{(P)}(\theta)$ will be filtered out by the distance filter $\left(f_{d}^{(P)}<0.16 \phi_{\max }^{(P)}\right)$. Hence, the only beam elements left by the distance filter $f_{d}^{(P)}$ defined in Eq.(4) are the ones closest to the component.

The dependency of the function $\delta^{(P)}$ to $\theta$ was chosen to account for the fact that beam elements at $\pm 45^{\circ}$ are closer to one another than elements at $0^{\circ}$ or $90^{\circ}$. This implies that for $\theta= \pm 45^{\circ}$, the bandwidth of the filter must be reduced compared to $\theta=0^{\circ}$, otherwise the model would be too stiff. 


\subsubsection{Location filter $f_{l}$}

The location filter $f_{l}$ cancels out elements that are not located within the two extremities of the component. Similarly to the distance filter, it depends on nodal filter functions that are evaluated at the end-nodes of each beam element. The expression of the location filter $f_{l}$ is as follows:

$f_{l}=\sqrt{f_{l_{\text {node }}}\left(d_{\|}\left(\mathrm{Q}_{i}\right)\right) \cdot f_{l_{\text {node }}}\left(d_{\|}\left(\mathrm{R}_{i}\right)\right)}$

where $d_{\|}$is the distance between each end-node of the $i$-th beam element and the perpendicular bisector $\mathcal{B}$ of the component, as illustrated in Figure 6a. The nodal location filter is defined as :

$f_{l_{\text {node }}}\left(d_{\|}\right)=\exp \left(\frac{-d_{\|}^{p} \cdot \ln (2)}{\left(k a+\frac{1}{2} L_{c}\right)^{p}}\right)$

where $L_{c}$ is the length of the component, $a$ the side of the square shell element, whilst $k$ and $p$ are numerical parameters (respectively, a coefficient defining the distance $k a$ proportionally to the element size and an exponent) that are specifically tuned for the filter to be effective. Exponent $p$ is chosen in the interval $20<p<100$. This ensures that $f_{l_{\text {node }}} \approx 1$ for any end-node that falls in-between the extremities of the component and $f_{l_{\text {node }}}=0$ outside. Consequently, the location filter does not interfere with the other filters. The transition from $f_{l_{\text {node }}}=1$ to $f_{l_{\text {node }}}=0$ is centered at a distance $k a$ from the extremities of the component, recommending $0<k<2.5$, as shown in Figure $6 \mathrm{~b}$.

\subsection{Multiple Components}

We have described so far the definition of the projection function $\phi^{(P)}$ for any beam element in the ground finite element mesh, in the case of the presence of a single component in the geometrical representation (i.e. one single straight stiffener). In order to accommodate more than one component on the surface, intersections and overlaps of components must be handled: for each component $c\left(c \in\left\{1, \ldots, N_{c}\right\}\right.$, where $N_{c}$ is the overall number of components in the geometrical representation), one can evaluate the resulting projection functions $\phi_{c}^{(P)}$ for all beam elements in the ground FE mesh, according to the procedure described in the previous section. Then, one has to fix a rule for the definition of the final value of the projection function $\phi^{(P)}$ for each beam element in order to build the structural representation of the stiffeners. In the literature, the value of $\phi^{(P)}$ of each beam element is determined either by summing the $N_{c}$ components' contributions $\phi_{c}^{(P)}$ (Norato et al. 2015) or by conserving the maximum components' contributions

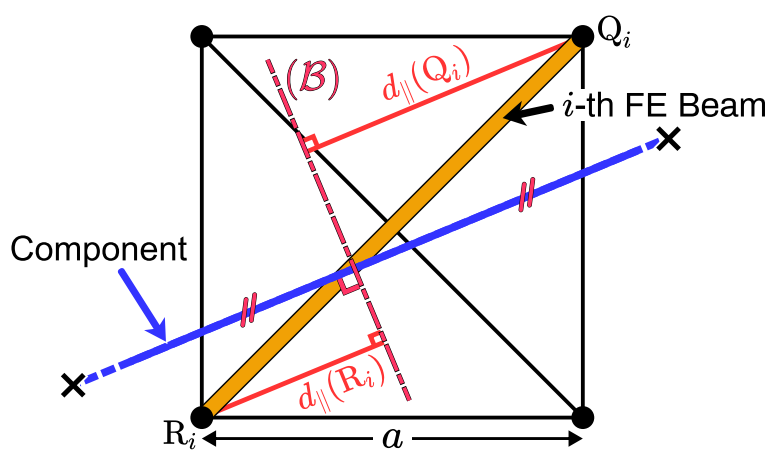

(a)

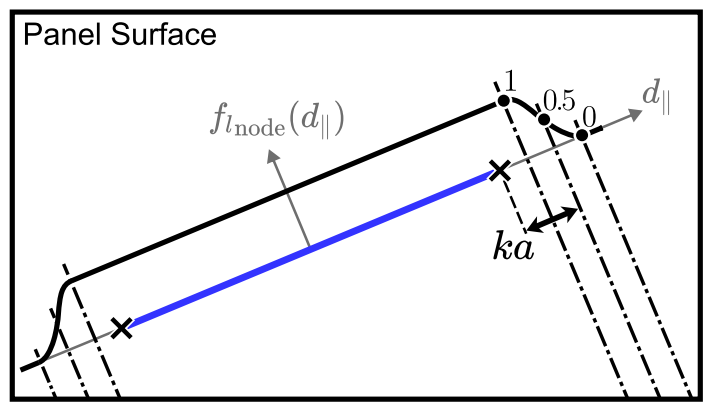

(b)

Fig. 6 (a) Variables of the nodal distance filter $f_{l_{\text {node }}}$. (b) Shape and contour lines of the nodal distance filter $f_{l_{\text {node }}}$ over the panel surface.

as in Zhang et al. (2016b) and Zhang et al. (2016a). In this paper, the latter formulation is used :

$\phi^{(P)}=\max _{c \in\left\{1, \ldots, N_{c}\right\}} \phi_{c}^{(P)}$

In the case of gradient-based optimization, the max function cannot be used directly as it is not differentiable. Consequently, it is approximated in this work by a $p$-norm :

$\phi^{(P)}=\left[\phi_{\min }^{p}+\left(1-\phi_{\min }^{p}\right) \sum_{c=1}^{N_{c}}\left(\phi_{c}^{(P)}\right)^{p}\right]^{\frac{1}{p}}$

where $\phi_{\text {min }}$ is introduced as a small positive lower bound to avoid an ill-posed analysis, as done in Zhang et al. (2016a). This prevents the beam elements from having null cross-sectional properties which cannot be handled by the FEA.

\section{Optimization Process}

The general form of the optimization problem solved in this article can be stated as:

$\min _{\{\mathbf{X}\}} F(\mathbf{X})$ subject to: $\left\{\begin{array}{l}\mathbf{X} \in \mathcal{D} \\ g_{i}(\mathbf{X}) \leq 0, i=\{1, \ldots, n\}\end{array}\right.$ 


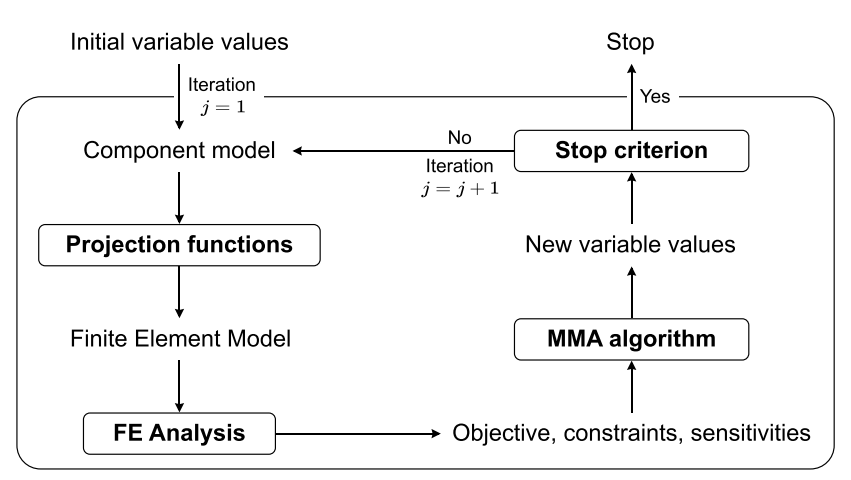

Fig. 7 Schematic overview of the stiffener location optimization process.

where $F$ is the objective function, $\mathbf{X}$ the vector of variables, which take values in the domain $\mathcal{D}$, and $g_{i}$ are constraint functions.

The objective $F$ and the constraints $g_{i}$ can be chosen among the available responses, either calculated analytically (e.g. mass) or output by a linear FE analysis (e.g. compliance, maximum displacements, critical buckling load, etc.). The optimization variables are the coordinates $\left(x_{1}^{1}, y_{1}^{1}, x_{2}^{1}, y_{2}^{1}, \ldots, x_{1}^{N_{c}}, y_{1}^{N_{c}}, x_{2}^{N_{c}}, y_{2}^{N_{c}}\right)$ of the two extremities of the $N_{c}$ components in the model. Their domain of variation $\mathcal{D}$ is bounded by the sides of the surface. The material and cross-sectional properties are identical for all components, and remain constant during the optimization, thus the object of the optimization is specifically the stiffening path.

The optimization process is illustrated in Figure 7 . The design variables define the geometry of the component model. At each iteration $j$, the components are projected on the structural model via the projection functions detailed in Section 2. A structural analysis outputs the values of the objective and constraint functions. The Method of Moving Asymptotes (MMA) (Svanberg 1987) is used to calculate the values of the design variables for the iteration $j+1$. The optimization process stops when either a maximum number of iterations $j_{\max }$ is attained, or a stagnation criterion on the maximum change of the design variables values from iterations $j-1$ to $j$ is fulfilled :

$\left\{\begin{array}{l}\max \left(\left|X_{j}-X_{j-1}\right|\right) \leq \varepsilon \\ j \leq j_{\max }\end{array}\right.$

where $\varepsilon$ is a small positive real value.

In order to use a gradient-based algorithm like the MMA, the sensitivities of the responses (both objective and constraints) with respect to the variables must be derived. This operation is usually expensive when it is done by finite differences. The advantage of the proposed method is that sensitivities are derived semianalytically, thus yielding lower computation costs. The process is similar to the one described in Deklerck and Abdalla (2016). The analytical part of the sensitivities is obtained by deriving the projection function (Eqs. (1) to (7)) and the stiffener assembly function (Eq.(9)). The output is the sensitivity of the cross-sectional properties of the beam elements with respect to the variables, i.e. $\partial P / \partial \boldsymbol{X}$. In the present work, the sensitivity of the response $R$ (i.e. compliance, in the present case) with respect to the cross-sectional properties of the beam elements is obtained using Altair optistruct. Details on the computation of the sensitivities in the software can be found in Altair Engineering (2019). Finally, by composition of functions, the full sensitivity can be obtained by :

$$
\begin{aligned}
\frac{\partial R}{\partial \boldsymbol{X}}= & \frac{\partial R}{\partial A} \cdot \frac{\partial A}{\partial \boldsymbol{X}}+\frac{\partial R}{\partial I_{y}} \cdot \frac{\partial I_{y}}{\partial \boldsymbol{X}}+\frac{\partial R}{\partial I_{z}} \cdot \frac{\partial I_{z}}{\partial \boldsymbol{X}} \\
& +\frac{\partial R}{\partial J} \cdot \frac{\partial J}{\partial \boldsymbol{X}}+\frac{\partial R}{\partial h} \cdot \frac{\partial h}{\partial \boldsymbol{X}}
\end{aligned}
$$

where $P=\left\{A, I_{y}, I_{z}, J, h\right\}$ are the properties of the beam elements belonging the ground structure, as defined in Eq.(1) Section 2.3, and $\boldsymbol{X}$ is the vector of the variables, which refer to the components representing the stiffeners to be optimised over the base panel.

\section{Numerical Calibration}

The objective of the calibration procedure is to tune the parameters of the projection functions defined in Section 2, in order to obtain a good agreement between the structural FE model or structural representation of the stiffener that is used for the optimization, and a reference FE model. The reference FE model is based on a fine conformal mesh of the skin and the components. Figure 8 shows a thickness-rendered view of the representation of the stiffener in the reference FE model and its structural representation based on the ground FE mesh, as used in the optimization process. In the reference FE model, the stiffener is represented as a single line of beam elements along the path defined by each component in the geometrical representation. The optimization model corresponds to the structural representation of the stiffener, i.e. an equivalent lattice structure based on the ground FE mesh, that is built as explained in the previous sections. The agreement between these two models is judged acceptable if the following requirements are satisfied:

i. the errors on the response values are limited: this is particularly necessary when the responses are used as constraints of the optimization; 
ii. the variations of the responses have the same monotonicity: this ensures that the search is driven towards areas of interest of the design space that are relevant in order to find the expected optimum;

iii. the localized nature of the component is conserved in the structural model.

The parameters $\phi_{\max }^{(P)}$ and functions $\delta^{(P)}$ are introduced in Eq.(5). Their values are specific to each crosssectional property $P=\left\{A, I_{y}, I_{z}, J, h\right\}$. The parameter $\phi_{\max }^{(P)}$ controls the maximum amplitude of the distributed cross-sectional property $P$, whilst the function $\delta^{(P)}$ controls the number of beam elements over which the cross-sectional property $P$ is distributed in the component width. Note that $\phi_{\max }^{(P)}$ and $\delta^{(P)}$ are not independent. For a given value of compliance, at a fixed angle $\theta$, if $\phi_{\max }^{(P)}$ is increased, the bandwidth of the filter must be reduced in order to maintain the same overall compliance value (i.e. $\delta^{(P)}(\theta)$ must be reduced), and vice versa. Using Eq.(5), the cross sectional properties are distributed on a total width of $4 a \delta^{(P)}$, centered on the axis of the component. To simplify the calibration procedure, we chose to use the same projection functions for all the inertias $I_{y}, I_{z}$ and $J$, i.e. $\phi^{I_{y}}=\phi^{I_{z}}=\phi^{J}=\phi^{I}$; separately, functions $\phi^{I}$ and $\phi^{h}$ are calibrated following the same procedure as for $\phi^{A}$. The same associations are made on the function $\delta^{(P)}$.

The reference cases are built using a square plate with a single stiffener. The plate is clamped along one edge and a uniaxial loading is applied to the opposite edge: two load cases are considered, a membrane (inplane uniform tension) and a bending (transversal uniform force) load, so that $\phi^{A}$ and $\phi^{I}$ can be calibrated separately. Two parametric studies are conducted by sweeping a stiffener over the plate: a parallel sweeping of a longitudinal stiffener $\left(\theta=0^{\circ}\right)$ over 5 positions separated by $a / 4$ (see Figure $9 \mathrm{a}$ ), and an angular sweeping of a single stiffener, passing through the center of the plate, from $\theta=0^{\circ}$ to $\theta=45^{\circ}$ by steps of $5^{\circ}$ (see Figure $9 \mathrm{~b})$. In order to obtain smooth variations of the structural response, a reference FE model of the stiffened plate is built in coincidence with each position of the stiffener in the sweeping parametric studies. The calibration is based on the comparison of global compliance between the optimization and reference FE models. Note that the cross-section of the stiffener used for the calibration must be the same as the one used for the optimization: changing the cross-sections will require a new calibration.

The calibration procedure of the area and inertia projection functions $\phi^{A}$ and $\phi^{I}$ can be stated as an optimization problem :

$$
\min _{\left\{\phi_{\max }^{(P)},\left\{\boldsymbol{\delta}^{(P)}\right\}\right\}} \sqrt{\sum_{k=1}^{K} \frac{\left(C_{k}-C_{k}^{\text {ref }}\right)^{2}}{K}}
$$$$
\text { subject to: } \frac{\partial\{\boldsymbol{C}\}}{\partial Z} \odot \frac{\partial\left\{\boldsymbol{C}^{\mathrm{ref}}\right\}}{\partial Z} \geqslant \mathbf{0}
$$

where $\{\boldsymbol{C}\}$ and $\left\{\boldsymbol{C}^{\text {ref }}\right\}$ are vectors respectively containing the compliance values of the structural and reference FE models, for all the $\mathrm{K}$ sweeping positions (position index $k, k \in\{1, \ldots, K\}) .\left\{\boldsymbol{\delta}^{(P)}\right\}$ are the discrete values of the function $\delta^{(P)}$ at each calibration angle $\theta$, $\odot$ is the element-wise product, $Z$ is the position of the stiffener in the parallel sweeping case. The objective function corresponds to the minimization of the Root Mean Square Error (RMSE) between the reference response and the response of the optimization model. The constraint imposes that both responses share the same monotonicity. The local nature of the stiffener is enforced by choosing the highest feasible value of $\phi_{\max }^{(P)}$, which corresponds to minimizing the bandwidth of the distance filter $f_{d}^{(P)}$. This satisfies the requirements (iiii) introduced at the beginning of the section.

The effects of the discrete nature of the ground structure on the monotonicity of the response is best observed by doing the parallel sweeping. If the bandwidth $4 a \delta^{(P)}(\theta)$ is too thin, the value of the compliance shows significant rises whenever the stiffener overlaps a beam element, followed by a drop-off at the next step, when the stiffener sits in-between two beam elements. This leads to oscillations in the value of the compliance, creating spurious local optima. On the other hand, if the bandwidth is too wide, the localized nature of the stiffener is compromised.

The optimization problem (13) is solved using a hierarchical approach: the first step of the calibration consists in finding the highest $\phi_{\max }^{(P)}$, associated with the thinnest bandwidth, that prevents oscillations from occurring in the parallel sweeping case. This is an iterative process, starting with $\phi_{\max }^{(P)}=1$. The value $\delta^{(P)}\left(0^{\circ}\right)$ that minimizes the RMSE in the $\theta=0^{\circ}$ configuration of the angular sweeping is found using a Newton-Raphson method. The constraint of the optimization problem (13) is then evaluated using the parallel sweeping. This process is repeated by gradually decreasing $\phi_{\max }^{(P)}$ until the constraint is satisfied. Once $\phi_{\max }^{(P)}$ is set, the NewtonRaphson method is applied to find the best value of $\delta^{(P)}$ for each $\theta$ value of the angular sweep.

Finally, the discrete set $\left\{\boldsymbol{\delta}^{(P)}\right\}$ is interpolated to define the continuous and derivable function $\delta^{(P)}(\theta)$ for $\theta \in\left[0^{\circ}, 45^{\circ}\right]$ (see Figure 10). By construction of the distance filter $f_{d}^{(P)}$, the function $\delta^{(P)}(\theta)$ is pair and periodic with a period $\pi$. In the following, a polyno- 


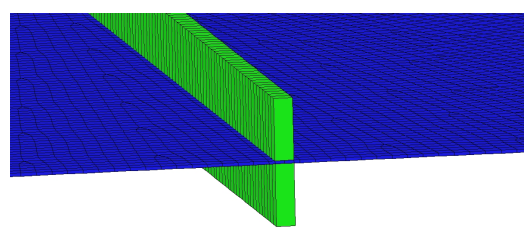

(a)

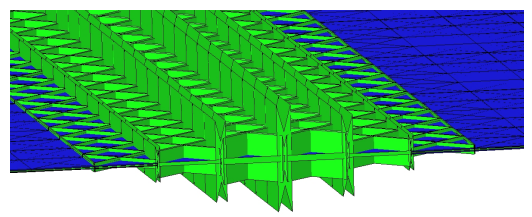

(b)

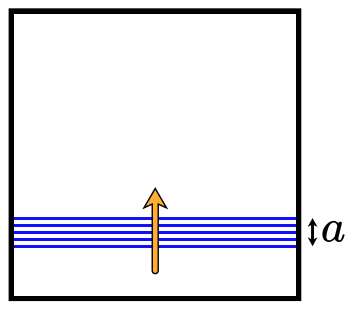

(a)

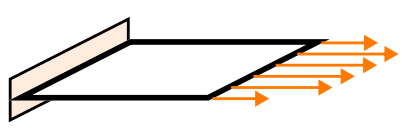

(c)

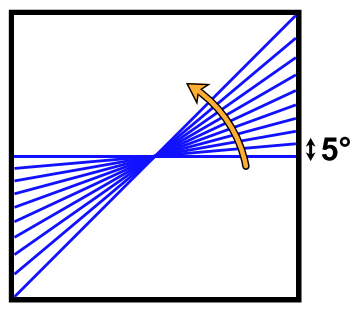

(b)

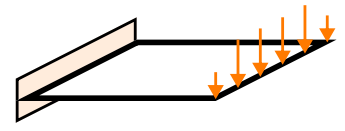

(d)

Fig. 9 Parallel sweep (a) and angular sweep (b) reference cases submitted either to a pure uniform tension (c) or a pure uniform bending (d) load case.

mial interpolation is used to define $\delta^{(P)}(\theta)$. Results of the calibration will be shown in the following section, which describes the considered cases of application for the optimization method.

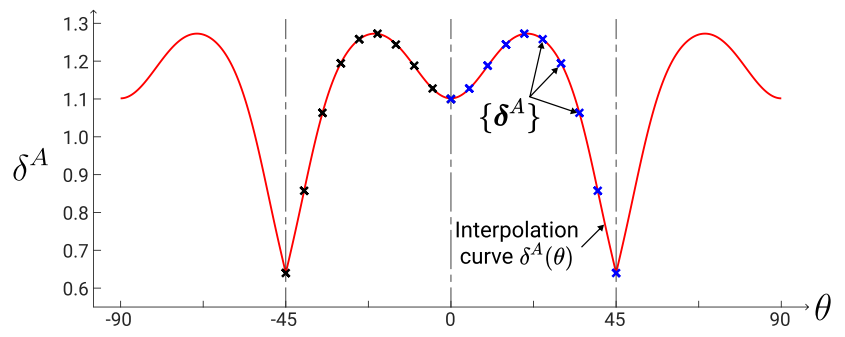

Fig. 10 Construction of the function $\delta^{A}(\theta)$ by a polynomial interpolation of the optimal values $\left\{\boldsymbol{\delta}^{A}\right\}$ deduced from the angular sweeping.

It is noteworthy that the obtained set of parameters $\left\{\phi^{(P)}, \boldsymbol{\delta}^{(P)}\right\}$ is specific to the cross-section property calibrated, and remains constant during the optimization. Nevertheless, using both a tension and a bending load case for the calibration procedure allows to reduce

Fig. 8 Thickness-rendered view of the reference FE model of a stiffener (a) and its associated structural model based on the ground FE mesh (b). the problem dependency. Furthermore, a mesh-size dependent parameter $a$ is introduced in the distance filter $f_{d}^{(P)}$ (Section 2). This allows some flexibility if the mesh size of the optimized panel is not the same as the mesh size of the calibration model.

Contrary to what is usually done in feature mapping methods which typically apply the projection on to the stiffness matrix, the parameters of the beam section (area, inertia) are projected independently. This allows to differentiate longitudinal tension compression behavior from bending behavior. Indeed, the corresponding terms in the stiffness matrix of the element do not present the same regularity with respect the cross-sectional properties. Tests were performed by projecting the stiffness matrix with either the projection function $\phi^{A}$ or $\phi^{I}$ and in this case, either the tension compression behavior or the bending behavior is accurate but not both at the same time. On the contrary, in the proposed method both behaviors are simultaneously accurate.

\section{Applications}

The proposed optimization method is first tested and validated on basic cases of plates with a clamped edge. It is then applied to cylindrical structures. The proposed optimization process is implemented in Matlab and the FE analyses are performed using Altair OPTISTRUCT. The optimization algorithm used is Svanberg's 2007 implementation of the MMA algorithm. The material properties, the stiffener cross-sections, the projection function calibration and the mesh size are identical for all the applications shown in this paper. The skin panels and the stiffeners are made of steel $\left(E=210 \mathrm{GPa}, \nu=0.3, \rho=7845 \mathrm{~kg} / \mathrm{m}^{3}\right)$. The crosssectional properties of the stiffeners are derived from a $6 \times 40 \mathrm{~mm}$ rectangular section with no section offset $(h=0 \mathrm{~mm})$. The results of the calibration of the projection functions (see Section 4) are presented in Table 1 . The table presents the values of $\phi_{\max }^{A}$ and $\phi_{\max }^{I}$. Here, $\delta^{A}(\theta)$ and $\delta^{I}(\theta)$ are defined as 12-th order sparse polynomials of the form $\delta^{(P)}(\theta)=\sum_{k=0}^{6} c_{2 k} \theta^{2 k}$, where $P=A$ or $P=I$ and $\theta$ in radians. The non-zero coefficients and monomials are given in Table 1 . The $p$-norm 
as in Eq.(9) is used with $p=8$ and its lower bound parameter set to $\phi_{\min }=1 \times 10^{-8}$. Finally, all the models of the following applications are meshed with square elements with an approximate size $a=20 \mathrm{~mm}$.

\subsection{Applications on planar surfaces}

The applications of this section focus on the minimization of the global compliance of stiffened plates. After considering two unconstrained optimizations with only two stiffeners, a mass constraint and component removal strategy are formulated enabling optimizations with a greater number of stiffeners.

\subsubsection{Unconstrained optimization}

The first application considered is a $1 \mathrm{~m} \times 1 \mathrm{~m} \times 1 \mathrm{~mm}$ thick square plate (the plate occupies the domain $[x, y] \in[0,1] \times[0,1]$ in the $\mathrm{x}-\mathrm{y}$ plane, see Figure 11$)$. The ground structure is made up of $50 \times 50$ shell elements and 10100 beam elements connecting all adjacent nodes, corresponding to an overall number of 15300 degrees of freedom. The plate is clamped at one edge $(x=0)$ and submitted to either an in-plane punctual load of $F_{y}=20 \mathrm{kN}$ or an out-of-plane punctual load of $F_{z}=200 \mathrm{~N}$ on the middle of its opposite side $(x=1$ and $y=0.5)$. The load is linearly distributed over five nodes to smooth the load introduction. The initialization of the stiffeners' distribution corresponds to two straight stiffeners forming a cross, centered on the plate (see Figure 11). The maximum number of iterations is set to $j_{\max }=50$ and the parameter of the stagnation criterion is set to $\varepsilon=0.006$. In order to use the MMA for solving this unconstrained problem, a dummy constraint is introduced, which is always satisfied (MMA only can handle constrained problems).

The results of the optimizations are shown in Figure 11. For the in-plane load case, the optimization converges in 23 iterations. The stiffest design is obtained at the final iteration and has a compliance of $4.67 \mathrm{~J}$ (66\% of the initial compliance). For the out-of-plane load case, the optimization converges in 34 iterations. The stiffest design is obtained at iteration 14 and has a compliance of $2.12 \mathrm{~J}$ (1.1\% of the initial compliance). The average iteration time is $30 \mathrm{~s}$ with $75 \%$ of the time occupied by the FE analysis : the optimization is performed on a standard laptop and the linear finite element analysis is run on a dedicated work station. This time may seem large relatively to the simplicity of the FE model, but this is due to the FEA sensitivity calculation done by OPTISTRUCT (4 CPU, $800 \mathrm{MB}$ RAM).
The resulting optimal positions of the stiffeners are consistent with the geometry and loading of the cases at study. The convergence towards the stiffest design is fast, and slows down over the last iterations during which the positions and lengths of the stiffeners do not evolve much. Note that the compliance values in the final iterations of the out-of-plane load case are less than $1 \%$ higher than the compliance of the stiffest design.

\subsubsection{Mass constrained optimization}

The second application aims at dealing with a greater number of stiffeners in the initial design, and possibly removing some of them during the course of the optimization. To create an incentive to removing stiffeners, the available quantity of material used to form stiffeners must be constrained. In the present study, this constraint is formulated as a maximum total mass of stiffeners. In the present study, a constraint $g(\boldsymbol{X})$ is applied on the total allowable mass of stiffeners $\bar{M}$, such that:

$g(\boldsymbol{X})=M-\bar{M}$

$M$ being the total mass of the stiffeners expressed as:

$M=\rho \sum_{c=1}^{N_{c}} A_{c} * L_{c}$

In Eq.(15), $A_{c}$ and $L_{c}$ are respectively the cross-sectional area and the length of the $c^{\text {th }}$ stiffener, and $\rho$ the density of the considered material. The advantage of this formulation is that the mass of the stiffeners corresponds exactly to the mass of a conformly meshed model. It also ensures a smoother variation of the mass with respect to changes in the stiffener's length and/or position, compared to the mass calculated from the structural model (overall mass of the projection of the stiffener over beams elements in the ground structure). Finally, the formulation of the mass in Eq.(15) implies that if two stiffeners are superimposed, the model will have a mass equal to that of two stiffeners but a global compliance of only one (this is the consequence of using the maximum function to assemble the stiffeners, see Section 2.4): this drives the optimizer to avoid overlapping stiffeners. Hence, in the present study, the only possibility for the optimizer to remove components is by shortening, i.e. reducing their size down to a very low value or eventually to zero. In order to stabilize the optimization, a stiffener removal condition is introduced : if the length of a stiffener is smaller than three times the mesh size for three consecutive iterations, the stiffener is canceled by simply deleting its variables.

The application considered is a $1.5 \mathrm{~m} \times 1 \mathrm{~m}$ plate (the plate occupies the domain $[x, y] \in[0,1.5] \times[0,1]$ 


\begin{tabular}{lcccccccc}
\hline Section property & $\phi_{\max }^{(P)}$ & $c_{12}$ & $c_{10}$ & $c_{8}$ & $c_{6}$ & $c_{4}$ & $c_{2}$ & $c_{0}$ \\
\hline Area $(P=A)$ & 0.4 & -146.43 & 314.15 & -259.38 & 108.12 & -27.502 & 3.5499 & 1.1016 \\
Inertia $(P=I)$ & 0.16 & 258.32 & -461.54 & 295.52 & -67.497 & -7.1133 & 4.1854 & 0.79449 \\
\hline
\end{tabular}

Table 1 Results of the calibration of the projection functions for the cross sectional area $(P=A)$ and the inertia $(P=I)$. Values of $\phi_{\max }^{(P)}$ and coefficients of the polynomial functions $\delta^{(P)}(\theta)$.
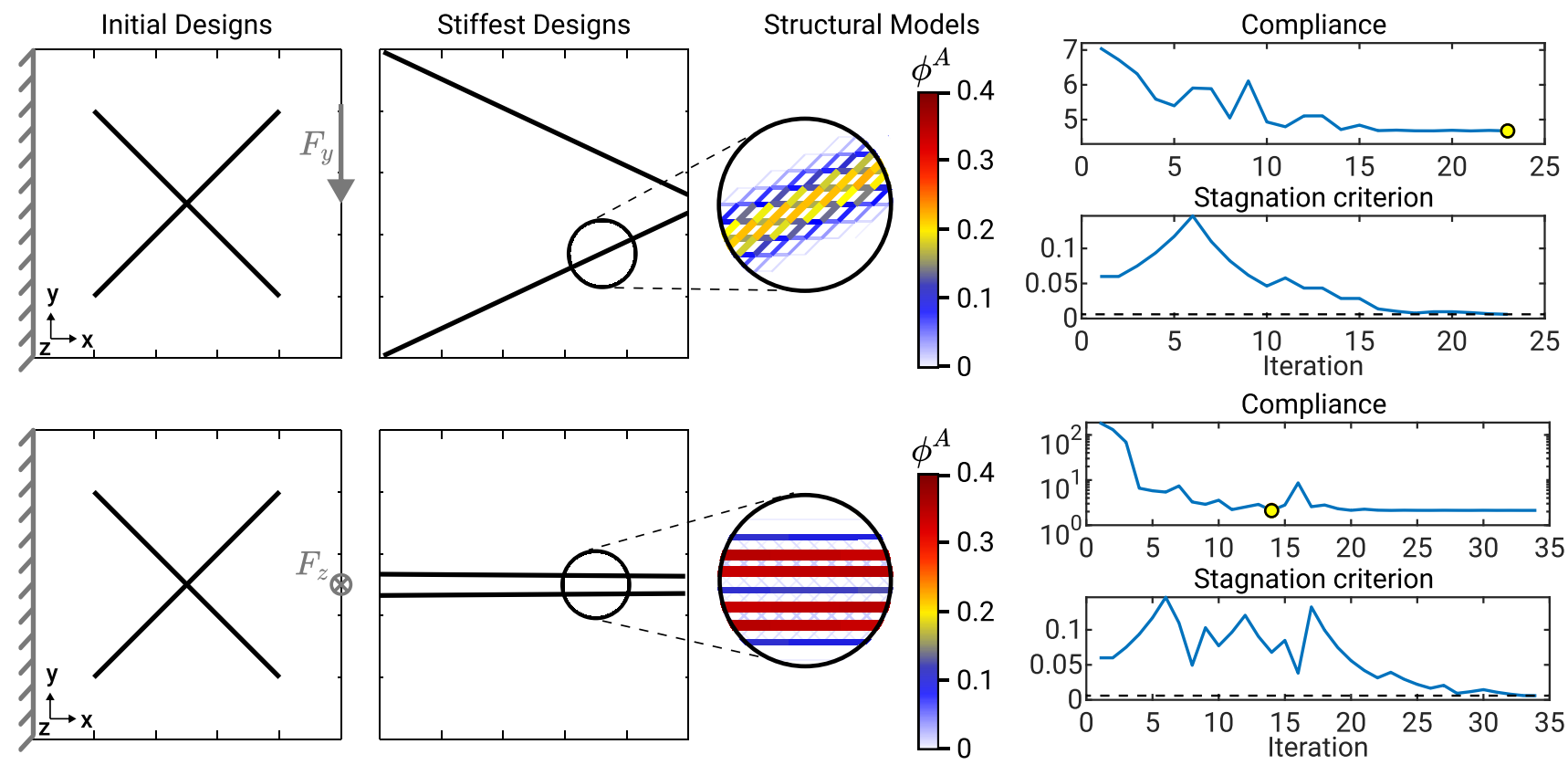

Fig. 11 Clamped plate unconstrained optimization with in-plane loading (top) and out-of-plane loading (bottom). From left to right: initial designs, stiffest designs (iteration 23 and 14 respectively), detailed views of the equivalent structural models, history of the compliance (stiffest design marked by yellow dot) and stagnation criteria.

in the x-y plane, see Figure 12), inspired by the well known cantilever problem on truss structures, as solved by Cao et al. (2018). In order to simulate a truss structure case, the thickness of the plate is set to $0.01 \mathrm{~mm}$, so that the plate stiffness is negligible with respect to the component stiffness. The plate is clamped at one edge $(x=0)$ and submitted to an in-plane punctual load of $F_{y}=10 \mathrm{kN}$ on the middle of its opposite side $(x=1.5$ and $y=0.5)$. The load is linearly distributed over three nodes to smooth the load introduction. The initialization of the stiffener distribution is made up of 16 components distributed in two lines of 4 crosses (see Figure 12). The mass constraint is set to $M<12 \mathrm{~kg}$, the normalized stagnation criterion is set to $\bar{\varepsilon}=1 \%$ of the variation domain of the variables and the maximum number of iterations is set to $j_{\max }=100$.

The results are presented in Figure 12. The optimization converges in 71 iterations. The stiffest feasible design is obtained at the final iteration of the optimization and has a compliance of $5.6 \mathrm{~J}$ for a stiffener mass of $M=11.99 \mathrm{~kg}$. Only 10 components remain out of the initial 16.
The resulting optimal component placements are coherent with the final topology of the cantilever beam obtained in Cao et al. (2018), even though the results differ slightly due to the limitation to a fixed component cross-section in the present case. Regarding the interpretation of the results, it seems that the final and stiffest design still contains components that are too short to have a significant mechanical contribution (two very short components are hidden at the very left corners, and two short components are visible on the right side of the structure). These components have not satisfied the component removal condition, nevertheless they should be ignored when interpreting the results. It can also be noted that the top and bottom components are slightly longer than expected as they extend beyond the intersections of components found near $x=1$. By zooming on the structural model, it can be seen that beam elements from the ground structure actually participate in a mechanical connection between the respective components after the joints $(x>1)$. This contributes to lowering the compliance of the structural model, however, these links would not exist in a con- 
formly meshed model. One can imagine that the parts of the components extending beyond the intersections could thus be removed in the interpretation.

\subsection{Applications on cylindrical surfaces}

The following applications consider cylindrical parts, closer to what can be found in space launcher structures. The projection method is adapted to deal with cylindrical surfaces and an unconstrained global compliance minimization is conducted on a cylinder with and without an access hatch. A mass-constrained optimization is then considered on the cylinder with an access hatch and compared to results obtained with a commercial implementation of the SIMP optimization.

\subsubsection{Parametrization of the components: from plates to cylinders}

Section 2 describes the parametrization of the components for a flat rectangular plate. The rectangular plate can be seen as an isoparametric representation of the surface of the structure into a Cartesian grid, which allows any developable structure to be represented in this way. In particular, the parametrization of the stiffener trajectory (position and length) at component-level can be extended to a cylinder with minor addition to the method, by projecting the surface of the cylinder onto the isoparametric rectangular plate. The projection of the surface of the cylinder in the isoparametric plane is a rectangular surface. The FE mesh is transformed accordingly.

However, one peculiar aspect of cylindrical structures is their periodicity (angle $\psi \in[0,2 \pi]$, see Figure 13), which must be accounted for in the parametrization. A stiffener can cross the cutting line $\psi=0$, from which the cylinder is developed, where $\psi$ is the radial component of the cylindrical coordinate system associated to the structure, and $\psi=0$ corresponds equally to $x=0$ and $x=1$ in the isoparametric plane. Thus, one single stiffener that crosses the cutting line $\psi=0$ on the cylinder must be split between the left $(x=0)$ and right $(x=1)$ sides of the isoparametric projection, resulting into two components represented over the rectangular domain. Moreover, we built the parametrization in order to account for the fact that a stiffener can extend over several turns on the cylinder. Since the rectangular projection corresponds to a single period of that gyration, then the rectangular surface is replicated $N_{r}$ times along the $x$-direction of the isogeometric plane and the boundaries for the $x$-coordinate of the components are increased accordingly. The projections of the components on the isoparametric mesh can be computed using the method described in Section 2. Then, the components can be brought back onto the initial rectangular mesh by considering for each beam element the maximal value of $\phi^{(P)}$ over its replicas:

$\phi^{(P)}=\max _{r \in\left\{1, \ldots, N_{r}\right\}} \phi_{r}^{(P)}$

where $\phi_{r}^{(P)}$ is the projection factor of the cross-sectional property $P$ of the $r$-th replica of the element. The max function is here also approximated by a $p$-norm, with $p=8$ (see Eq.(9)).

\subsubsection{Unconstrained optimizations}

The proposed optimization method is now applied to a cylindrical structure, either with a stiffened access hatch or without (referred hereafter as regular cylinder). The cylinder has a perimeter of $p_{\text {cyl }}=2 \mathrm{~m}$, a height of $h_{\text {cyl }}=1 \mathrm{~m}$ and a skin thickness of $1 \mathrm{~mm}$. The hatch itself is modeled by deleting shell and beam elements from the ground structure. However, a contour of beam elements is kept to form the frame of the hatch, which is fixed during the optimization. The corresponding beam elements have the same cross-sectional properties as the stiffeners. The hatch has a width of $160 \mathrm{~mm}$, a height of $320 \mathrm{~mm}$ and is placed $-90^{\circ}$ away from the direction of the load $F$ (see Figure 14). Regarding boundary conditions, the nodes of the bottom edge are clamped. The nodes of the top edge of the cylinder are rigidly linked by RBE2 elements to a central master node on the top of the cylinder, where a shearing load $F_{x}=100 \mathrm{kN}$ is applied. To initialize the optimization process, four stiffeners are placed in two cross-like patterns. The maximum number of iterations is set to $j_{\max }=50$ and the parameter of the stagnation criterion is set to $\varepsilon=0.036$.

The results of the optimizations are shown in Figure 14. The optimization on the regular cylinder converges in 18 iterations and the stiffest design is obtained at iteration 15 . The optimization on the cylinder with an access hatch converges in 18 iterations and the stiffest design is obtained at iteration 14 . The convergence profiles are similar to the ones presented in Figure 11, and the designs tend to oscillate between closely similar solutions over the last iterations. In the figure, the minimal compliance solutions found are marked with a yellow point on the compliance curve. For both optimizations, it does not coincide with the final iteration. However, the compliance values after the minimal compliance points vary by a magnitude inferior to $2 \%$. These variations are sufficiently small to consider that all the positions attained after the minimal compliance points are equivalent. 

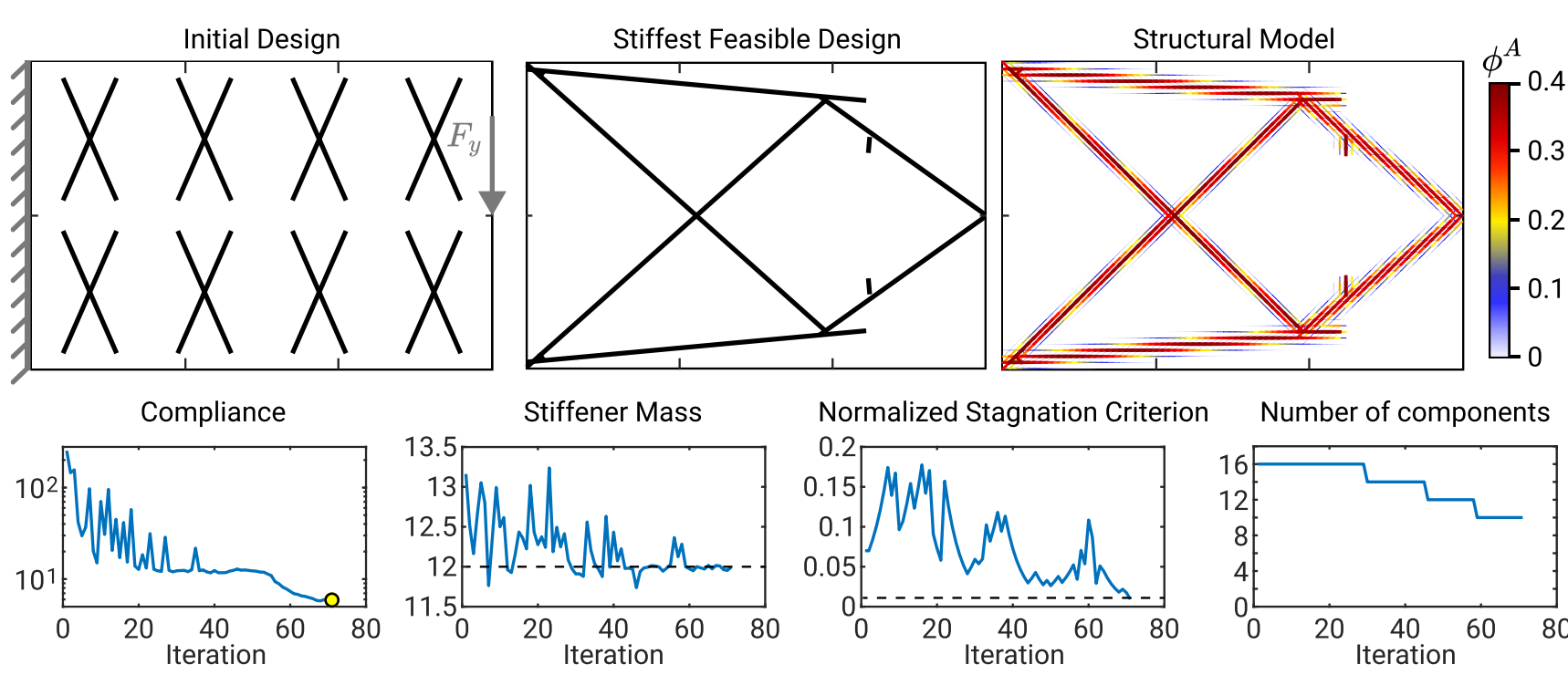

Number of components

Fig. 12 Mass constrained compliance minimization of a cantilever plate of negligible thickness, similar to a truss structure. Top, from left to right: geometrical model of the initial design with boundary conditions and stiffest feasible design with its corresponding structural model. Bottom: history of compliance (stiffest feasible design marked by yellow dot), total stiffener mass $M$, normalized stagnation criteria $\bar{\varepsilon}$ and number of components.

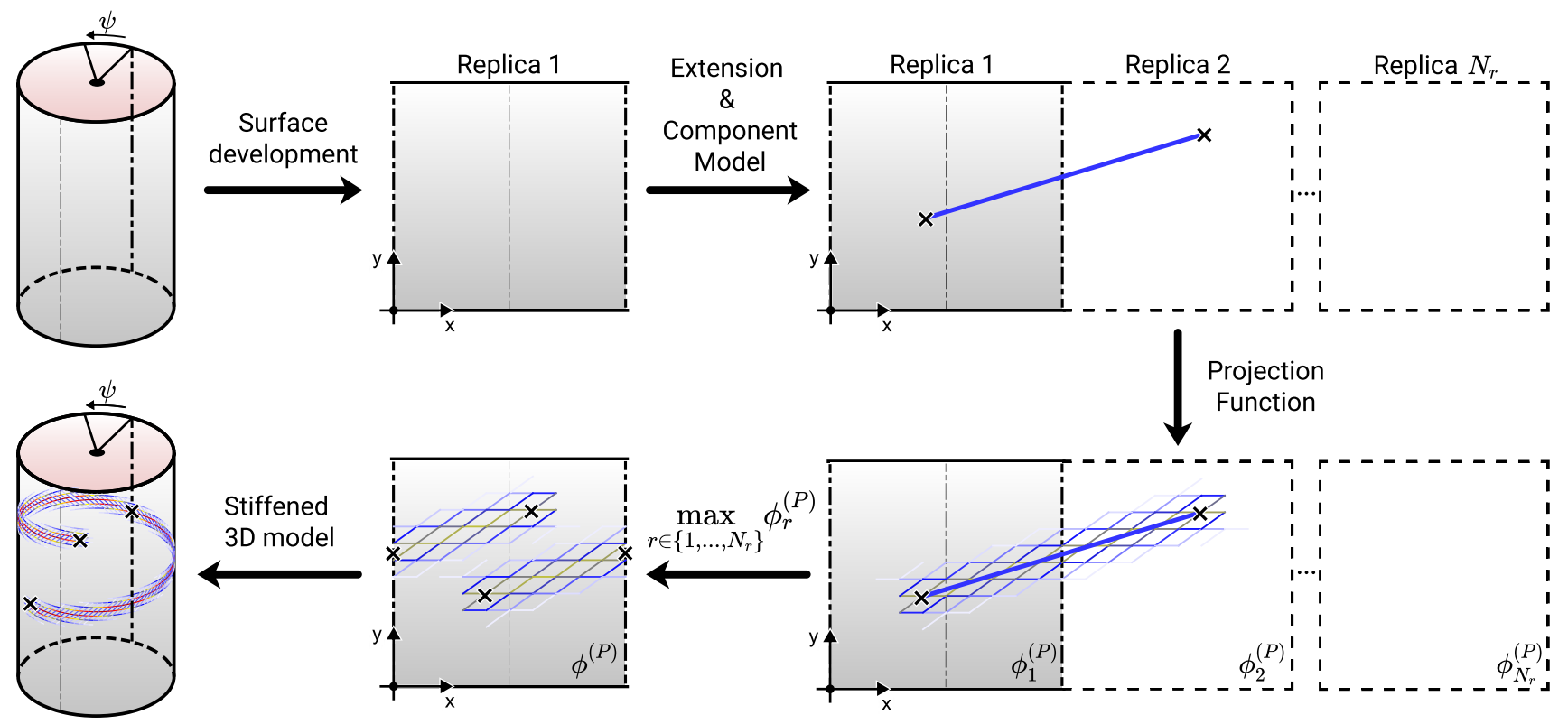

Fig. 13 Projecting a stiffener onto a three-dimensional cylindrical surface.

To evaluate the accuracy of the compliance values computed using the optimization model, a reference conformal FE model is built for the regular cylinder in the stiffening configurations of iterations 15 and 18 , using the same modeling principles as in Section 4. In the stiffening configuration of iteration 15 , the compliance computed with the optimization model $(103.5 \mathrm{~J})$ is $0.78 \%$ higher than the compliance of the reference model $(102.7 \mathrm{~J})$. In the stiffening configuration of itera- tion 18 , the optimization value $(103.6 \mathrm{~J})$ is $1.17 \%$ higher than the reference $(102.4 \mathrm{~J})$. Note that the compliance of the reference models differ by less than $0.3 \%$ between the two stiffening configurations at iterations 15 and 18 , which can thus be considered equivalent, similarly to the results on the optimization model. The same comparisons are made on the cylinder with an access hatch in the stiffening configuration of iteration 14. The compliance computed with the reference model $(123.9 \mathrm{~J})$ is 

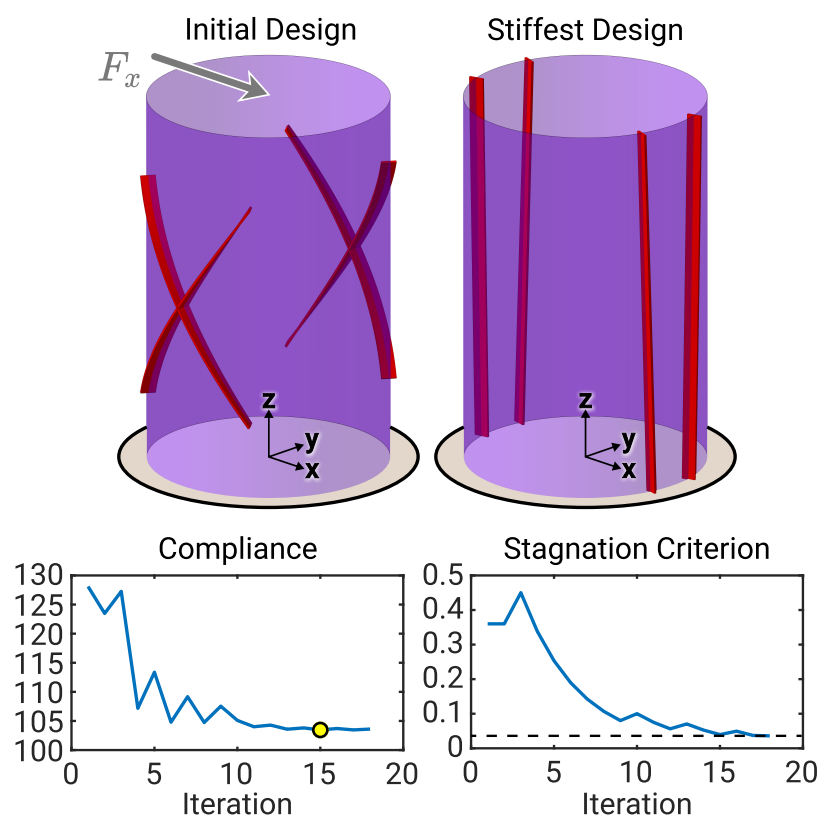
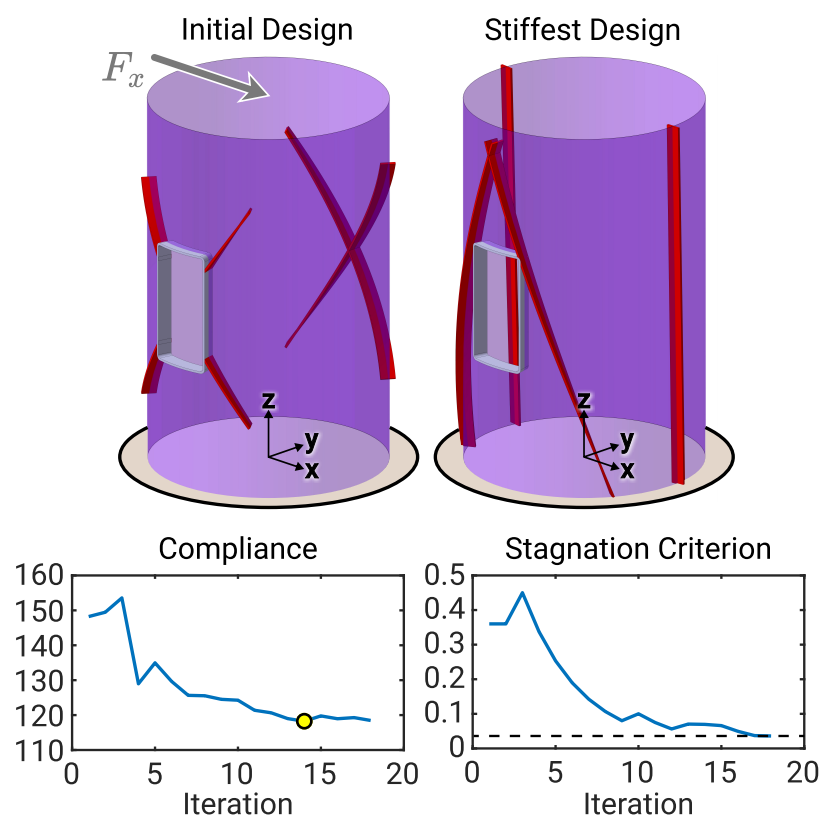

Fig. 14 Unconstrained compliance minimization of a cylinder submitted to a shearing load. Top: CAD views of the stiffener layouts on the initial and stiffest designs for a regular cylinder (left, iteration 15) and for a cylinder with an access hatch (right, iteration 14). Bottom: corresponding histories of the compliance (stiffest designs marked by yellow dot) and stagnation criteria.

$4.6 \%$ higher than the compliance of the optimization model in the same stiffening configuration (118.2 J). These comparative elements globally confirm a good agreement between the geometrical (component) and the structural (equivalent FE model) representations, thanks to the calibration phase.

Nonetheless, these results highlight the fact that the method is capable of finding the optimal placement of the stiffeners, with a relatively small tolerance on the stiffener positions. The precision of the optimization can be limited by several factors, namely the quality of the projection functions, the ground structure mesh and the settings of the optimization algorithms. Whilst the latter point is out of the scope of the paper, the quality of the projection functions is intimately related to the ground mesh and it is driven by the ratio between the finite element size and the bandwidth of the filter $f_{d}$. As long as the aspect ratio of the shell elements is kept close to one, the calibration procedure detailed in Section 4 proves to be effective at minimizing the bias introduced by the projection step for a given FE mesh.

\subsubsection{Mass constrained optimization}

In this last application, the global compliance of the cylinder with an access hatch (see previous section) is minimized under a constraint on the maximum allowable mass of the stiffeners (not including the access hatch stiffening members). The stiffener mass is derived in the same way as in section 5.1.2, and the proposed stiffener removal strategy is applied. The mass constraint is set to $M<10 \mathrm{~kg}$ in the first run and $M<20$ $\mathrm{kg}$ in the second one. The geometries and boundary condition on the cylinder are the same as those of the previous section. The initial design is made of 24 components, disposed in four columns of 3 crosses (see Figure 15). However, only the 12 stiffeners on the positive $\mathrm{x}$ values are optimized, the rest of the components being obtained by symmetry. The normalized stagnation criteria are set to $\bar{\varepsilon}=1 \%$ of the variables' variation domain and the maximum number of iterations are set to $j_{\max }=100$.

Figure 15 illustrates the results of these two optimizations. The optimization with the $10 \mathrm{~kg}$ mass constraint converges in 65 iterations. The stiffest feasible design, indicated by the yellow points in the compliance graph, is achieved at iteration 64 with a compliance of $102.9 \mathrm{~J}$ (28 \% compliance reduction from the initial design), a total stiffener mass of $9.99 \mathrm{~kg}$ and with 16 stiffeners left out of the 24 initial. The optimization with the $20 \mathrm{~kg}$ mass constraint converges in 77 iterations. The stiffest feasible design is achieved at iteration 76 with a compliance of $80.2 \mathrm{~J}$ (44\% compliance reduction from the initial design), a total stiffener mass of $19.98 \mathrm{~kg}$ and 20 stiffeners left. The equivalent conformal $\mathrm{FE}$ model of this iteration has a compliance of 

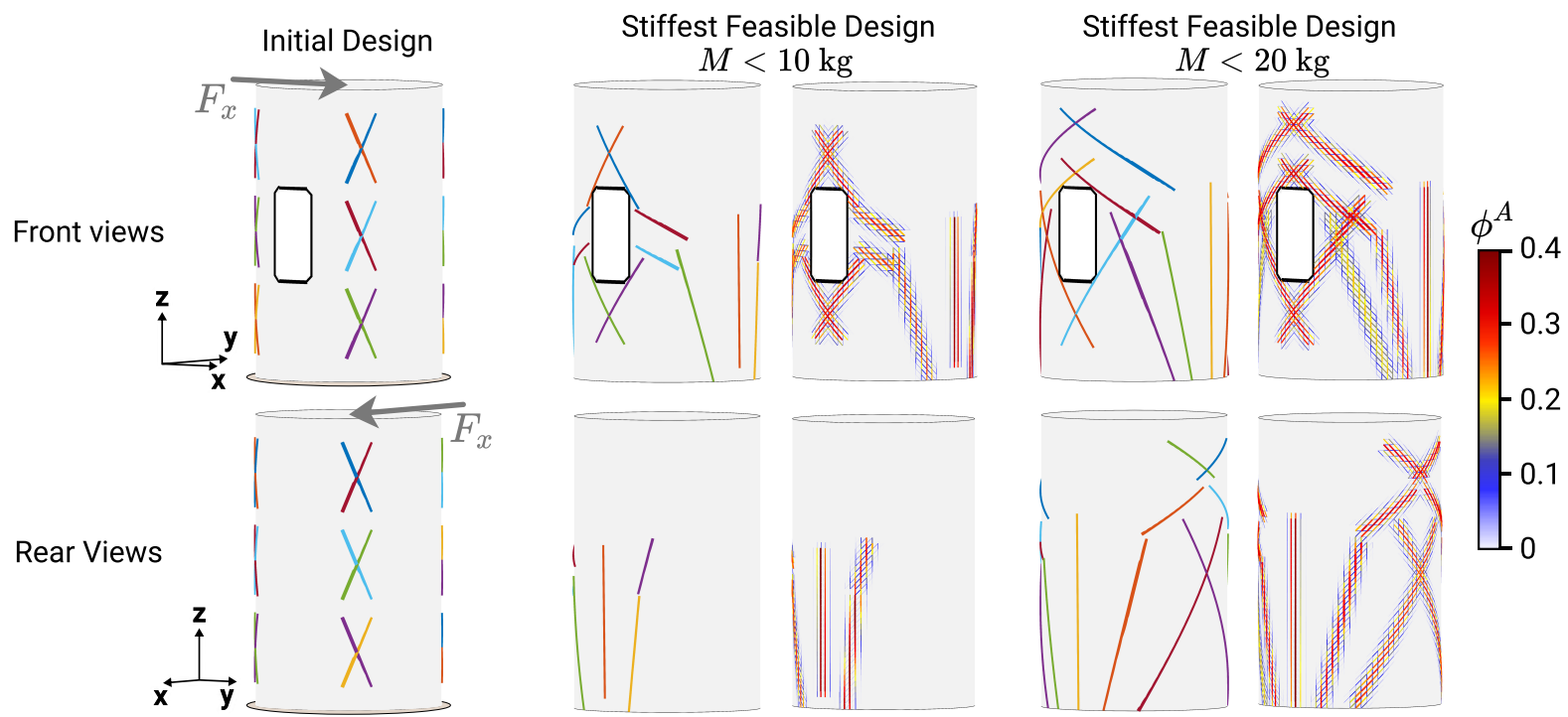

Compliance

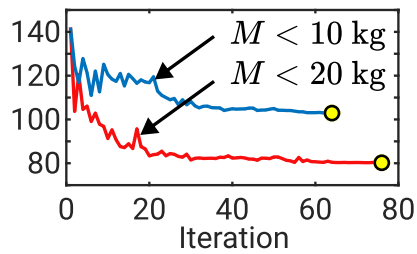

Stiffener Mass $M$

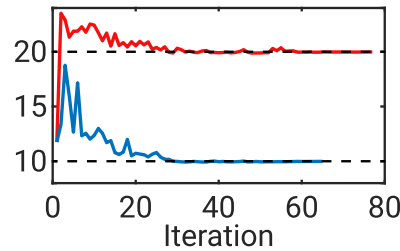

Normalized Stagnation Criteria

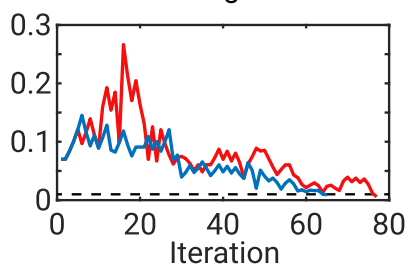

Number of components

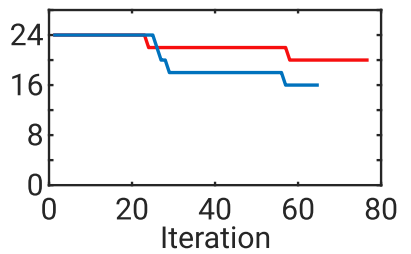

Fig. 15 Mass constrained compliance minimization of a cylinder with access hatch. Top: front and rear views of the geometrical models of the initial design and of the stiffest feasible designs with their corresponding structural models for a stiffener mass constraint of $M<10 \mathrm{~kg}$ (iteration 64) and $M<20 \mathrm{~kg}$ (iteration 76). Bottom: histories of compliance (stiffest feasible designs marked by yellow dot), total stiffener mass $M$, normalized stagnation criteria $\bar{\varepsilon}$ and number of components.

$86.4 \mathrm{~J}$ and a stiffener mass of $19.98 \mathrm{~kg}$. In both cases, the optimization demonstrates a good convergence profile on the compliance and stiffener mass values. The effectiveness of the mass constraint and of the component removal strategy is also demonstrated. Finally, the optimization model here also demonstrates a good accuracy in the evaluation of the compliance and mass values of its equivalent conformal $\mathrm{FE}$ model (-8 \% error on compliance, $0 \%$ error on mass).

\subsubsection{SIMP optimizations of the cylinder with access hatch}

In the latter case of the cylinder with an access hatch, the positions of the stiffeners minimizing the compliance of the structure can not be easily inferred by common sense, as opposed to the previous applications. In order to assess the relevance of the stiffest feasible stiffening structures obtained (see Figure 15), the latter will be compared to solutions obtained via SIMP topological optimizations carried out using OPTISTRUCT.
The first topological optimization was conducted using the same ground structure (made of beam and shell elements) as the structural FE model of the cylinder with access hatch. The load case and boundary conditions are also the same. The variables are defined as the material densities of the beam elements. These beam elements have the same material and cross-sectional properties as the components in the above examples. The objective is to minimize the compliance for a maximum allowed total mass of $25 \mathrm{~kg}$, chosen close to the total mass of our optimal solution $(27 \mathrm{~kg})$. The result of this optimization, presented in Figure 16a, does not allow to determine a straightforward stiffening path. Furthermore the compliance of the model $(520 \mathrm{~J})$ is only slightly reduced compared to the un-stiffened cylinder $(677 \mathrm{~J})$. This behavior is caused by the low volume fraction of beam elements allowed: the mass of the allowed design domain ( $8 \mathrm{~kg}$ of beam elements) represents only $0.9 \%$ of the total mass of the design domain $(889 \mathrm{~kg}$ if all the densities of the beam elements are set to 1). Such a low constraint on the volume fraction usually makes the convergence of the SIMP method to an acceptable 
solution very difficult. By relaxing the constraint on the maximum total mass to $100 \mathrm{~kg}$, the design domain is widened, and the solution obtained makes use of much more beam elements (see Figure 16b). Consequently, the compliance $(240 \mathrm{~J})$ is better reduced compared to the previous model, however, the solution remains difficult to interpret in terms of stiffening paths. Nonetheless, the areas reinforced in Figures 15 and 16 seem consistent (upper and lower vertices of the rectangular hatch and areas located at $\pm 90^{\circ}$ of the hatch along the circumference of the cylinder). These observations highlight the advantages of the proposed component method: by controlling the set of beam elements, efficient designs $(102.9 \mathrm{~J}, 27 \mathrm{~kg}$ or $80.2 \mathrm{~J}, 37 \mathrm{~kg}$ ) with clearly defined stiffeners are obtained directly, even if a very low volume fraction is used. Moreover, the compliance and mass of the engineered final design (i.e. the reference FE model) are accurately predicted.

To gain further insight on the relevance of our optimal solutions, a topological optimization using shell elements as ground structure was used, with the aim of identifying potential stiffening paths. The variables are here the material density in each element, treated by the SIMP method. The shell elements of the ground structure are defined with a thickness of $12 \mathrm{~mm}$, which multiplied by their side length of $a=20 \mathrm{~mm}$, equals the cross-sectional area of our components. By imposing a maximum mass constraint of $80 \mathrm{~kg}$, the result in Figure 17 is obtained (similarly to the SIMP on beam elements, a mass constraint set too low does not allow to infer stiffening paths). In this figure, clear regions of high material densities can be identified as potential stiffening paths, even though medium density zones persist, where the stiffening path is not evident. Similarities can be observed between this solution and our optimal solutions. Regarding the area around the hatch, the main stiffening path is accurately found using the component method proposed in this paper and a similar density distribution can be inferred from the topology optimization results. A thinner secondary stiffening path to the sides of the hatch can be identified from Figure 17 which seems to be common to the stiffening paths obtained with our method and the $20 \mathrm{~kg}$ mass constraint. Concerning the area diametrically opposite of the hatch the topology optimization suggests curved stiffeners with bigger dimensions at the bottom of the cylinder. Large regions of intermediate density values remain, making it difficult to infer discrete stiffening paths from this result. Comparing the model obtained with the SIMP to the ones obtained with our method, it can be seen that the base of the cylinder are similarly reinforced at a location $\pm 90^{\circ}$ away from the hatch (which is consistent with both models of Figures 15).
The model obtained with our method and the $10 \mathrm{~kg}$ constraint has very few stiffeners on the rear side of the cylinder but seems to adequately reproduce the bottom of these main stiffening paths. However, the stiffeners do not extend to the top of the cylinder because of the mass constraint. On the other hand, the model obtained with the $20 \mathrm{~kg}$ mass constraint is overall in better agreement with the SIMP optimization, as shown by the curvature and by the length of the stiffeners, extending from bottom to top. However, the top parts of the stiffening structures are slightly different: with our proposed method, the resulting stiffening paths cross at the top part of the cylinder contrary to the SIMP result. Noticing that the proposed method has also determined clear stiffening paths in the region where the SIMP method converged to intermediate densities, this could justify the slight differences observed in the stiffening paths. From these comparisons, the optimal stiffening structure found with our method seems to be relevant when compared to stiffening structures deduced from SIMP optimizations. Furthermore, it allows a straightforward interpretation of the stiffening structure in terms of a feasible engineering design, especially in zones where the SIMP optimization converges to intermediate densities.

\section{Conclusions}

This paper presents a method to optimize the layout of stiffeners on large shell structures. The proposed method can be seen as an extension of the component-based methods (Guo et al. 2014; Zhang et al. 2018, 2017; Norato et al. 2015; Zhang and Norato 2017; Coniglio et al. 2020; Bai and Zuo 2020): stiffeners, assimilated to components, are projected as sets of beam elements on a ground structure meshed using structural elements, i.e. shells and beams. By doing so, the stiffener, which is intrinsically a beam, is represented as an equivalent lattice in the optimization process. The projection method allows the components to move freely over the surface, without any remeshing operation. Furthermore, semi-analytical sensitivities can be derived by using a gradient-based cost-effective optimizer. A calibration procedure is proposed for the projection functions, which enables to minimize the discrepancy between the optimization model and a reference FE model. Three compliance minimization examples are given, showing the efficiency and accuracy of the method. The applications also show that the method is adapted to large cylindrical parts that may contain holes.

From the aforementioned elements, this method is adapted for the pre-sizing in compliance of large cylindrical shell structures, giving results that can be in- 


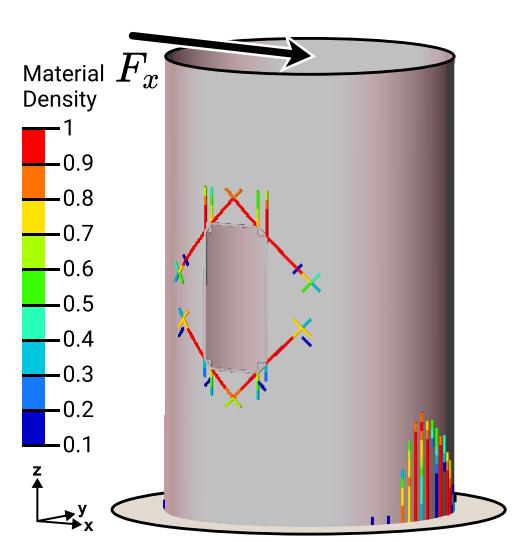

(a)

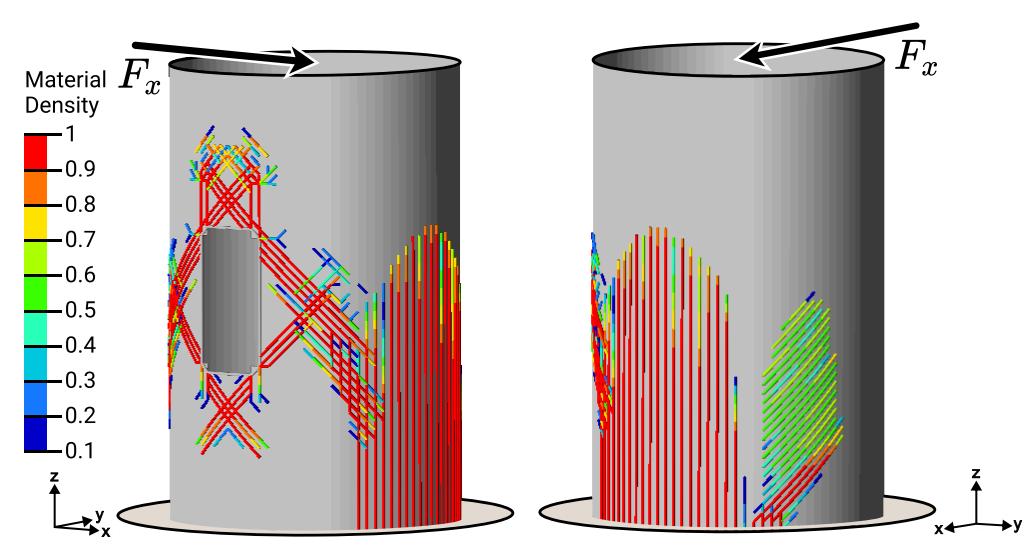

(b)

Fig. 16 Result of the SIMP compliance minimization of a cylinder with access hatch with a given maximum total mass of (a) $25 \mathrm{~kg}$ and (b) $100 \mathrm{~kg}$ : optimization of the material densities of beam elements (same ground structure as in the case of section 5.2.2, Figure 14).
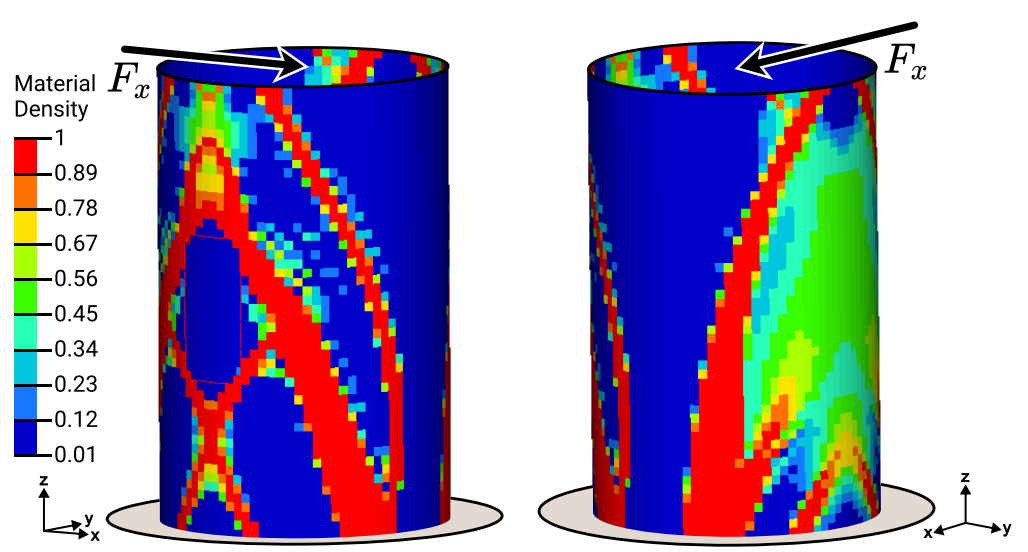

Fig. 17 SIMP topology optimization of a cylinder with access hatch with a given maximum total mass of $80 \mathrm{~kg}$ : optimization of the material density on a mesh of shell elements.

terpreted as feasible engineering designs. Further work should allow to extend the method to other structural responses, such as buckling loads, by verifying the accuracy of the responses of the optimization model. A further axis of development would be to consider other stiffener cross-sectional sizes and shapes, as well as curvilinear stiffening paths, and eventually integrate them as variables of the optimization.

\section{Conflict of interest}

The authors declare that they have no conflict of interest.

\section{Replication of results}

The current work is part of a confidential project, hence the developed codes cannot be disclosed at present. However, the method is open and transparent, and all the necessary information to reproduce the results has been mentioned.

\section{References}

Afonso S, Sienz J, Belblidia F (2005) Structural optimization strategies for simple and integrally stiffened plates and shells. Engineering Computations 22(4):429-452, doi:10.1108/02644400510598769

Altair Engineering (2019) Altair Engineering,OptiStruct User Guide

Bai J, Zuo W (2020) Hollow structural design in topology optimization via moving morphable component method. Structural and Multidisciplinary Optimization 61(1):187205, doi:10.1007/s00158-019-02353-0

Bedair O (2009) Analysis and Limit State Design of Stiffened Plates and Shells: A World View. Applied Mechanics Reviews 62(2):020801, doi:10.1115/1.3077137

Cao M, Ma H, Wei P (2018) A modified stiffness spreading method for layout optimization of truss structures. Acta Mechanica Sinica 34(6):1072-1083, doi:10.1007/s10409018-0776-x

Coniglio S, Morlier J, Gogu C, Amargier R (2020) Generalized Geometry Projection: A Unified Approach for Ge- 
ometric Feature Based Topology Optimization. Archives of Computational Methods in Engineering 27:1573-1610, doi:10.1007/s11831-019-09362-8

Deklerck M, Abdalla MM (2016) Optimization of stiffened panels using a combination of FEM and a predictorcorrector interior point method. Master Thesis, TU Delft

Ding X, Yamazaki K (2005) Adaptive growth technique of stiffener layout pattern for plate and shell structures to achieve minimum compliance. Engineering Optimization 37(3):259-276, doi:10.1080/0305215512331328231

Dugré A (2014) A design process using topology optimization applied to flat pressurized stiffened panels. PhD Thesis, Ecole Polytechnique de Montréal, Montréal, Canada

Guo X, Zhang W, Zhong W (2014) Doing Topology Optimization Explicitly and Geometrically-A New Moving Morphable Components Based Framework. Journal of Applied Mechanics 81(8):081009, doi:10.1115/1.4027609

Gurdal Z, Gendron G (1993) Optimal design of geodesically stiffened composite cylindrical shells. Composites Engineering 3(12):1131-1147, doi:10.1016/09619526(93)90070-Z

Hirschler T, Bouclier R, Duval A, Elguedj T, Morlier J (2019) The embedded isogeometric Kirchhoff-Love shell: From design to shape optimization of nonconforming stiffened multipatch structures. Computer Methods in Applied Mechanics and Engineering 349:774797, doi:10.1016/j.cma.2019.02.042

Jaunky N (1995) Buckling analysis and optimum design of multidirectionally stiffened composite curved panel. PhD Dissertation, Old Dominion University, Norfolk, Virginia

Kapania R, Li J, Kapoor H (2005) Optimal Design of Unitized Panels with Curvilinear Stiffeners. In: AIAA 5th ATIO and 16th Lighter-Than-Air Sys Tech. and Balloon Systems Conferences, American Institute of Aeronautics and Astronautics, Arlington, Virginia, vol 3, pp 17081737, doi:10.2514/6.2005-7482

Lam Y, Santhikumar S (2003) Automated rib location and optimization for plate structures. Structural and Multidisciplinary Optimization 25(1):35-45, doi:10.1007/s00158-002-0270-7

Li B, Hong J, Wang Z, Liu Z (2013) An Innovative Layout Design Methodology for Stiffened Plate/Shell Structures by Material Increasing Criterion. Journal of Engineering Materials and Technology 135(2):021012, doi:10.1115/1.4023781

Li B, Ge L, Hong J (2017a) An intelligent computational approach for design optimization of stiffened engineering structures. International Journal of Precision Engineering and Manufacturing 18(7):1005-1012, doi:10.1007/s12541017-0118-0

Li B, Hong J, Liu Z (2017b) A novel topology optimization method of welded box-beam structures motivated by lowcarbon manufacturing concerns. Journal of Cleaner Production 142:2792-2803, doi:10.1016/j.jclepro.2016.10.189

Li B, Liu H, Yang Z, Zhang J (2019) Stiffness design of plate/shell structures by evolutionary topology optimization. Thin-Walled Structures 141:232-250, doi:10.1016/j.tws.2019.04.012

Li W, Zheng A, You L, Yang X, Zhang J, Liu L (2017c) Rib-reinforced Shell Structure. Computer Graphics Forum 36(7):15-27, doi:10.1111/cgf.13268

Liu D, Lohse-Busch H, Toropov V, Hühne C, Armani U (2016) Detailed design of a lattice composite fuselage structure by a mixed optimization method. Engineering Optimization 48(10):1707-1720, doi:10.1080/0305215X.2015.1125262
Liu S, Li Q, Chen W, Hu R, Tong L (2015) H-DGTP-a Heaviside-function based directional growth topology parameterization for design optimization of stiffener layout and height of thin-walled structures. Structural and Multidisciplinary Optimization 52(5):903-913, doi:10.1007/s00158-015-1281-5

Luo J, Gea HC (1998) A systematic topology optimization approach for optimal stiffener design. Structural Optimization 16(4):280-288

Maes VK, Pavlov L, Simonian SMS (2019) An efficient semi-automated optimisation approach for (gridstiffened) composite structures: Application to Ariane 6 Interstage. Composite Structures 209:1042-1049, doi:10.1016/j.compstruct.2016.02.082

Merino J, Patzelt A, Steinacher A, Windisch M, Heinrich G, Forster R, Bauer C (2017) Ariane 6 - Tanks and structures for the new european launcher. In: Deutscher Luft- Und Raumfahrtkongress, Munich, p 450255

Mulani S, Locatelli D, Kapania R (2011) Grid-Stiffened Panel Optimization Using Curvilinear Stiffeners. In: 52nd AIAA/ASME/ASCE/AHS/ASC Structures, Structural Dynamics and Materials Conference, American Institute of Aeronautics and Astronautics, Denver, Colorado, doi:10.2514/6.2011-1895

Mulani SB, Slemp WCH, Kapania RK (2013) EBF3PanelOpt: An optimization framework for curvilinear blade-stiffened panels. Thin-Walled Structures 63:13-26, doi:10.1016/j.tws.2012.09.008

Niemann S, Kolesnikov B, Lohse-Busch H, Hühne C, Querin OM, Toropov VV, Liu D (2013) The use of topology optimisation in the conceptual design of next generation lattice composite aircraft fuselage structures. The Aeronautical Journal 117(1197):1139-1154, doi:10.1017/S0001924000008745

Norato JA, Bell BK, Tortorelli DA (2015) A geometry projection method for continuum-based topology optimization with discrete elements. Computer Methods in Applied Mechanics and Engineering 293:306-327, doi:10.1016/j.cma.2015.05.005

Shi S, Sun Z, Ren M, Chen H, Hu X (2013) Buckling resistance of grid-stiffened carbon-fiber thin-shell structures. Composites Part B: Engineering 45(1):888-896, doi:10.1016/j.compositesb.2012.09.052

Sun Z, Cui R, Cui T, Liu C, Shi S, Guo X (2020) An Optimization Approach for Stiffener Layout of Composite Stiffened Panels Based on Moving Morphable Components (MMCs). Acta Mechanica Solida Sinica doi:10.1007/s10338-020-00161-4

Svanberg K (1987) The method of moving asymptotes-a new method for structural optimization. International Journal for Numerical Methods in Engineering 24(2):359-373, doi:10.1002/nme.1620240207

Szczepanik M, Burczyński T (2012) Swarm optimization of stiffeners locations in 2-D structures. Bulletin of the Polish Academy of Sciences: Technical Sciences 60(2):241246, doi:10.2478/v10175-012-0032-7

Vasiliev V, Barynin V, Rasin A (2001) Anisogrid lattice structures - survey of development and application. Composite Structures 54(2-3):361-370, doi:10.1016/S02638223(01)00111-8

Wang D, Abdalla MM, Zhang W (2017) Buckling optimization design of curved stiffeners for grid-stiffened composite structures. Composite Structures 159:656-666, doi:10.1016/j.compstruct.2016.10.013

Wang D, Abdalla MM, Wang ZP, Su Z (2018) Streamline stiffener path optimization (SSPO) for embedded stiff- 
ener layout design of non-uniform curved grid-stiffened composite (NCGC) structures. Computer Methods in Applied Mechanics and Engineering 344(1):1021-1050, doi:10.1016/j.cma.2018.09.013

Wei P, Ma H, Wang MY (2014) The stiffness spreading method for layout optimization of truss structures. Structural and Multidisciplinary Optimization 49(4):667-682, doi:10.1007/s00158-013-1005-7

Wein F, Dunning PD, Norato JA (2020) A review on featuremapping methods for structural optimization. Structural and Multidisciplinary Optimization 62(4):15971638, doi:10.1007/s00158-020-02649-6

Xue K, Li YX, Wang WY (2012) VenationLike Rib Layout Design in Plate under Bending Loads. Journal of Biomimetics, Biomaterials and Tissue Engineering 13(1):31-40, doi:10.4028/www.scientific.net/JBBTE.13.31

Zhang S, Norato JA (2017) Optimal Design of Panel Reinforcements With Ribs Made of Plates. Journal of Mechanical Design 139(8):081403, doi:10.1115/1.4036999

Zhang S, Norato JA, Gain AL, Lyu N (2016a) A geometry projection method for the topology optimization of plate structures. Structural and Multidisciplinary Optimization 54(5):1173-1190, doi:10.1007/s00158-016-1466-6

Zhang S, Gain AL, Norato JA (2020a) Adaptive mesh refinement for topology optimization with discrete geometric components. Computer Methods in Applied Mechanics and Engineering 364:112930, doi:10.1016/j.cma.2020.112930

Zhang W, Yuan J, Zhang J, Guo X (2016b) A new topology optimization approach based on Moving Morphable Components (MMC) and the ersatz material model. Structural and Multidisciplinary Optimization 53(6):12431260, doi:10.1007/s00158-015-1372-3

Zhang W, Li D, Yuan J, Song J, Guo X (2017) A new threedimensional topology optimization method based on moving morphable components (MMCs). Computational Mechanics 59(4):647-665, doi:10.1007/s00466-016-1365-0

Zhang W, Liu Y, Du Z, Zhu Y, Guo X (2018) A Moving Morphable Component Based Topology Optimization Approach for Rib-Stiffened Structures Considering Buckling Constraints. Journal of Mechanical Design, Transactions of the ASME 140(11):111404, doi:10.1115/1.4041052

Zhang W, Jiang S, Liu C, Li D, Kang P, Youn SK, Guo X (2020b) Stress-related topology optimization of shell structures using IGA/TSA-based Moving Morphable Void (MMV) approach. Computer Methods in Applied Mechanics and Engineering 366:113036, doi:10.1016/j.cma.2020.113036

Zhang W, Li D, Kang P, Guo X, Youn SK (2020c) Explicit topology optimization using IGA-based moving morphable void (MMV) approach. Computer Methods in Applied Mechanics and Engineering 360:112685, doi:10.1016/j.cma.2019.112685

Zhao W, Singh K, Kapania RK (2019) Thermal Buckling Analysis and Optimization of Curvilinearly Stiffened Plates with Variable Angle Tow Laminates. Journal of Spacecraft and Rockets 56(4):1189-1204, doi:10.2514/1.A34378 